Published in final edited form as:

Chem Mater. 2017 October 10; 29(19): 8269-8281. doi:10.1021/acs.chemmater.7b02567.

\title{
Target-or-Clear Zirconium-89 Labeled Silica Nanoparticles for Enhanced Cancer-Directed Uptake in Melanoma: A Comparison of Radiolabeling Strategies
}

\author{
Feng Chen ${ }^{\dagger, \uparrow}$, Kai Ma ${ }^{\ddagger}, \uparrow$, Li Zhang $^{\dagger}$, Brian Madajewski ${ }^{\dagger}$, Pat Zanzonico", Sonia Sequeira ${ }^{\perp}$, \\ Mithat Gonen\#, Ulrich Wiesner ${ }^{*}, \neq$, and Michelle S. Bradbury ${ }^{*},, \S$ \\ †Department of Radiology, Sloan Kettering Institute for Cancer Research, New York, New York \\ 10065, United States \\ ‡Department of Materials Science \& Engineering, Cornell University, Ithaca, New York 14853, \\ United States \\ \$Molecular Pharmacology Program, Sloan Kettering Institute for Cancer Research, New York, \\ New York 10065, United States \\ "Department of Medical Physics, Sloan Kettering Institute for Cancer Research, New York, New \\ York 10065, United States \\ ${ }^{\perp}$ Research and Technology Management, Sloan Kettering Institute for Cancer Research, New \\ York, New York 10065, United States \\ \#Department of Epidemiology and Biostatistics, Sloan Kettering Institute for Cancer Research, \\ New York, New York 10065, United States
}

\section{Abstract}

\footnotetext{
*Corresponding Authors: bradburm@mskcc.org, ubw1@cornell.edu.

IAuthor Contributions

F.C. and K.M. contributed equally to the work, and M.B. and U.W. contributed equally to the work.

Supporting Information

The Supporting Information is available free of charge on the ACS Publications website at DOI: 10.1021/acs.chemmater.7b02567. Tables of previously reported renally excreted nano-particles, decay properties of widely used PET isotopes, estimation of number of ${ }^{89} \mathrm{Zr}$ per $\mathrm{C}^{\prime}$ dots, organ uptake details, radiation dosimetry, figures of nat $\mathrm{Zr}$ number estimation, PET imaging of ${ }^{89} \mathrm{Zr}$-DFOcRGDY-PEG-C ${ }^{\prime}$ dots (using GSH and APTES as linkers), PD-10 elution profiles, mouse plasma uptake details, MIP images of free ${ }^{89}$ r-oxalate in mouse, effect of EDTA challenge, MIP PET images of tumor-bearing mice, and biodistribution studies (PDF) First 1 hour Dynamic distribution video of mouse injected with chelator-free ${ }^{89} \mathrm{Zr}$ labeled cRGDY-PEG-C ${ }^{\prime}$ dots (AVI) First 1 hour Dynamic distribution video of mouse injected with chelator-based ${ }^{89} \mathrm{Zr}$ labeled cRGDY-PEG-C' ${ }^{\prime}$ dots (AVI) ORCID

Feng Chen: 0000-0001-6495-1030

Kai Ma: 0000-0003-4415-6894

Ulrich Wiesner: 0000-0001-6934-3755

Michelle S. Bradbury: 0000-0003-3147-4391

Author Contributions

Product preparation was performed by F.C. and K.M.; experimental design was done by F.C., K.M., P.Z., U.W., and M.S.B., and data acquisition was done by F.C., K.M., L.Z.. Data analysis and interpretation was done by F.C., K.M., L.Z., P.Z., U.W., and M.S.B., and manuscript preparation was done by F.C., M.S.B., K.M., P.Z., and U.W. All authors approved the final version of the manuscript.

Notes

The authors declare no competing financial interest.
} 
Designing a nanomaterials platform with high target-to-background ratios has long been one of the major challenges in the field of nanomedicine. Here, we introduce a "target-or-clear" multifunctional nanoparticle platform that demonstrates high tumor-targeting efficiency and retention while minimizing off-target effects. Encouraged by the favorable preclinical and clinical pharmacokinetic profiles derived after fine-tuning surface chemical properties of radioiodinated $\left({ }^{124} \mathrm{I}, t_{1 / 2}=100.2 \mathrm{~h}\right)$ ultrasmall cRGDY-conjugated fluorescent silica nanoparticles (C dots), we sought to investigate how the biological properties of these radioconjugates could be influenced by the conjugation of radiometals such as zirconium- $89\left({ }^{89} \mathrm{Zr}, t_{1 / 2}=78.4 \mathrm{~h}\right)$ using two different strategies: chelator-free and chelator-based radiolabeling. The attachment of ${ }^{89} \mathrm{Zr}$ to newer, surface-aminated, integrin-targeting $\mathrm{C}^{\prime}$ dots using a two-pot synthesis approach led to favorable pharmacokinetics and clearance profiles as well as high tumor uptake and target-to-background ratios in human melanoma models relative to biological controls while maintaining particle sizes below the effective renal glomerular filtration size cutoff $<10 \mathrm{~nm}$. Nanoconjugates were also characterized in terms of their radiostability and plasma residence half-lives. Our ${ }^{89} \mathrm{Zr}$-labeled ultrasmall hybrid organic-inorganic particle is a clinically promising positron emission tomography tracer offering radiobiological properties suitable for enhanced molecularly targeted cancer imaging applications.

\section{Graphical abstract}

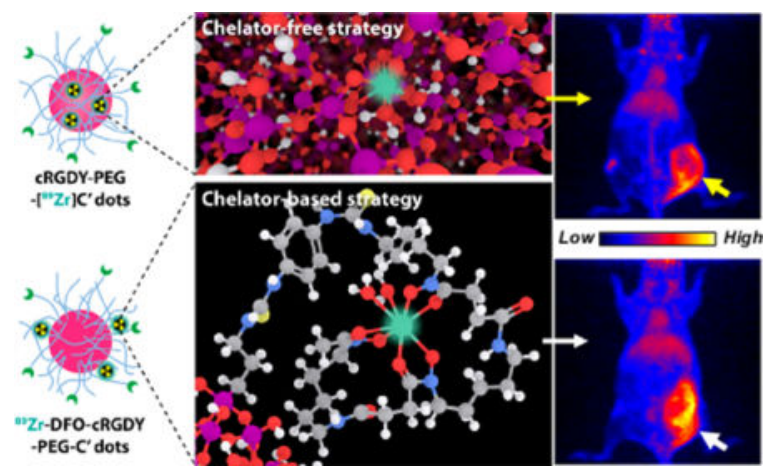

\section{INTRODUCTION}

Despite the promising preclinical research results of various types of solid (or inorganicbased) nanomaterials in small animals, ${ }^{1}$ very few of these platforms have progressed to firstinhuman clinical trials after more than 3 decades. ${ }^{2,3}$ In addition to manufacturing and regulatory challenges, rapidly rising clinical trial costs, and the increasing complexity of trial designs,${ }^{4}$ many existing nanomaterials show limited in vivo specific targeting efficacy ${ }^{5}$ and high liver accumulation rates ${ }^{6}$ (i.e., $30-99 \%$ of administered particles from the bloodstream), which are major hurdles that need to be addressed. For nanomaterials with a hydrodynamic (HD) size larger than $10 \mathrm{~nm}$, even with the protection of stealth polymers (e.g., polyethylene glycol [PEG]) and functionalized with tumor-homing ligands (e.g., peptides or antibodies), it is still extremely common to see predominant reticuloendothelial system (RES) (i.e., liver and spleen) uptake, tumor-to-liver activity concentration ratios less than 1 , and relatively low tumor-to-background (i.e., blood or muscle) ratios. ${ }^{6}$ High RES uptake also raises long-term in vivo toxicity concerns due to extremely slow and generally 
unpredictable hepatobiliary clearance rates from liver, with resulting delays in obtaining Investigational New Drug (IND) approval from the United States Food and Drug Administration (FDA). ${ }^{7}$ Therefore, even though larger-sized (i.e., $>10 \mathrm{~nm}$ ) solid nanomaterials have the advantage of significantly enhanced drug-loading capacity relative to their sub-10 nm sized counterparts, clinical translation of such materials may be hindered by low tumor targeting efficacy and high off-target (i.e., liver) accumulations associated with dose-limiting toxicity. 5,6

Fast renal clearance, relatively short blood circulation half-lives (ranging from several minutes to several hours), and low RES uptake (on the order of 5\% ID/g or less) represent defining biological features for ultrasmall (sub-10 nm) renally clearable nanoparticles (Table S1). ${ }^{8-17}$ Although suitable PEGylation techniques have been developed to improve the blood circulation half-life (up to $>10 \mathrm{~h}$ ) of such platforms, ${ }^{12,18}$ the ability to precisely control physicochemical properties, including surface ligand number, in a manner that facilitates bulk renal clearance while preserving tumor specific targeting capabilities, has long posed a significant challenge to the field. At present, and to the best of our knowledge, ultrasmall dye-encapsulating, $a_{\mathrm{v}} \beta_{3}$ integrin-targeting cyclic(arginine-glycine-aspartic acidD-tyrosine-cysteine) (cRGDY) peptide carrying and PEG-functionalized fluorescent coreshell silica nano-particles (also known as Cornell dots or cRGDY-PEG-C dots) are congruent with the foregoing description of highly desirable characteristics that are achievable for such nanoscale materials. ${ }^{16,17,19,20}$ Next-generation Cornell prime dots synthesized in waterbased environments, ${ }^{21}$ cRGDY-PEG-C' ${ }^{\prime}$ dots, are currently being utilized to study modulations in biological responses ${ }^{22}$ and as an iodine-124- $\left({ }^{124} \mathrm{I}, t_{1 / 2}=100.2 \mathrm{~h}\right)$ labeled, PET-optical platform for preclinical oncological indications ${ }^{23,24}$ and early phase clinical trials (NCT01266096 and NCT02106598).

Having a physical half-life comparable to that of ${ }^{124} \mathrm{I}$, zirconium-89 $\left({ }^{89} \mathrm{Zr}, t_{1 / 2}=78.4 \mathrm{~h}\right)$ is now a widely used positron-emitting radioisotope (Table S2) in preclinical studies ${ }^{25-27}$ and clinical trials. ${ }^{28}$ Moreover, ${ }^{89} \mathrm{Zr}$ has a much lower mean $\beta^{+}$energy (396 vs $820 \mathrm{keV}$ ), which may improve positron emission tomography (PET) spatial resolution. In contrast to radioiodine, which is prone to dehalogenation after cellular uptake (the extent to which depends on the nature of the chelator), $89 \mathrm{Zr}$ has been reported to residualize stably within cells after internalization, ${ }^{29}$ underscoring its potential to enhance targeted particle accumulations and target-to-background ratios. Herein, we investigate and compare chelatorbased and chelator-free radiolabeling strategies for attaching surface radiometals (i.e., ${ }^{89} \mathrm{Zr}$ ) to the water-based synthetic product, cRGDY-PEG-C ${ }^{\prime}$ dots. ${ }^{21}$ We sought to determine whether (1) chelator-free radiolabeling procedures, previously applied to larger size (porous and nonporous) silica particles, ${ }^{30,31}$ could be successfully extended to particle sizes below $10 \mathrm{~nm}$ and (2) resulting ${ }^{89} \mathrm{Zr}$-labeled peptide- and PEG-functionalized $\mathrm{C}^{\prime}$ dots (or cRGDYPEG-C ${ }^{\prime}$ dots) yielded high targeted uptake and target-to-background ratios in wellestablished integrin-expressing melanoma models while maintaining sub-10 nm sizes to facilitate renal excretion. Results of these findings could also inform development of a targeted radiotherapeutic platform by substitution of the diagnostic for a therapeutic radiolabel such as lutetium-177. 
The chelator-free strategy was achieved by ${ }^{89} \mathrm{Zr}$ labeling of the intrinsic deprotonated silanol groups (i.e., $-\mathrm{Si}-\mathrm{O}^{-}$) on the surface and within micropores of each particle at elevated temperature $\left(75^{\circ} \mathrm{C}, \mathrm{pH} 8\right.$ Scheme 1a). A traditional chelator-based ${ }^{89} \mathrm{Zr}$ labeling technique $\left(37^{\circ} \mathrm{C}, \mathrm{pH} 7.5\right)$ was also developed by carefully controlling the surface density of the selected chelator (i.e., p-SCN-Bn-deferoxamine or DFO-NCS) to maximize specific activity and radiochemical yields while maintaining the renal clearance property (Scheme 1b). Radiolabeled nanoconjugates were extensively characterized in terms of their radiostability, pharmacokinetic, clearance and dosimetry profiles, as well as their tumor targeting and target-to-background ratios. To the best of our knowledge, this is the first-of-its-kind ${ }^{89} \mathrm{Zr}$ labeled and renally clearable targeted organic-inorganic hybrid particle for dual-modality PET-optical imaging. On the basis of its favorable biological properties, including extended blood circulation half-life $(\sim 15 \mathrm{~h})$, high tumor targeting uptake $(>10 \% \mathrm{ID} / \mathrm{g})$, renal clearance (>60\% ID within 1-2 days), low liver accumulation ( $\sim 5 \% \mathrm{ID} / \mathrm{g}$ ), and high tumor-tobackground ratios (tumor:muscle $>9$; tumor:liver $>2$ ), this platform is a clinically promising diagnostic imaging tool for cancer-specific detection and localization in patients with melanoma while offering the potential to be further adapted as a targeted radiotherapeutic probe for treating disease.

\section{RESULTS AND DISCUSSION}

\section{Chelator-Free Zirconium-89 Radiolabeling of cRGDY-PEG-C' Dots}

Nanoparticle-based chelator-free radiolabeling has emerged as a novel intrinsic radiolabeling technique in the last several years, ${ }^{32}$ especially for radioisotopes (e.g., arsenic-72 [ ${ }^{72} \mathrm{As}, t_{1 / 2}$ $=26 \mathrm{~h}],{ }^{33,34}$ germanium- $69\left[{ }^{69} \mathrm{Ge}, t_{1 / 2}=39.1 \mathrm{~h}\right],{ }^{35}$ and titanium- $45\left[{ }^{45} \mathrm{Ti}, t_{1 / 2}=3.8 \mathrm{~h}\right]{ }^{36}$ ), for which suitable chelators are not currently available. Developing a chelator-free radiolabeling technique for ultrasmall renal clearable nano-particles is of particular interest because the introduction of additional surface modification steps may increase the particle's hydrodynamic radius and, in turn, reduce or eliminate renal clearance while promoting high liver uptake. Due to the presence of the intrinsic silanol groups $(-\mathrm{Si}-\mathrm{OH})$ on the surface or in micropores of each nanoparticle, ${ }^{37}$ silica is known to be one of the most versatile nanoplatforms for successful chelator-free labeling using a variety of radiometals, including ${ }^{89} \mathrm{Zr}^{30,31}$ The mechanism of labeling is thought to be due to strong interactions between a hard Lewis acid (i.e., radiometal of ${ }^{89} \mathrm{Zr}^{4+}$ ) and a hard Lewis base (i.e., deprotonated silanol groups, $-\mathrm{Si}-\mathrm{O}^{-}$, on silica surfaces). ${ }^{30}$ Although a large part of the outer surface silanol groups have been quenched after the surface PEGylation step using PEGsilane, ${ }^{18}$ we hypothesized that internal silanol groups from each microporous $\mathrm{C}^{\prime}$ dot are still accessible for the chelator-free ${ }^{89} \mathrm{Zr}$ labeling.

To that end, we radio-labeled cRGDY-PEG-C ${ }^{\prime}$ dots using ${ }^{89} \mathrm{Zr}^{4+}$ via the chelator-free strategy. $C^{\prime}$ dots were synthesized using a previously reported protocol. ${ }^{21}$ Near-infrared fluorescent $\mathrm{Cy} 5$ dyes were covalently encapsulated into the silica matrix of $\mathrm{C}^{\prime}$ dots, endowing $C^{\prime}$ dots with fluorescent properties; cancer targeting cRGDY peptides were then covalently attached to the outer surface of the $\mathrm{C}^{\prime}$ dots during PEGylation, allowing for improved tumor targeting. The resulting cRGDY-PEG-C $\mathrm{C}^{\prime}$ dots were purified and subjected to quality control analysis (Figure 1). The gel permeation chromatography (GPC) elugram of 
the purified cRGDY-PEG-C ${ }^{\prime}$ dot showed a single peak at around 9 min, corresponding to $\mathrm{C}^{\prime}$ dot nanoparticles (Figure 1a). The peak was well fit by a single Gaussian distribution, suggesting no detectable impurities and narrow particle size distributions (Figure 1a). The average hydrodynamic diameter of the purified cRGDY-PEG-C ${ }^{\prime}$ dots was $6.4 \pm 0.2 \mathrm{~nm}$ (Figure 1b), as measured by fluorescence correlation spectroscopy (FCS) and consistent with transmission electron microscopy (TEM) observations (Figure 1a). In addition to particle size, FCS also provides the particle concentration, which we used to estimate the number of functional groups per particle, including dyes, targeting peptides, and ${ }^{89} \mathrm{Zr}$ radioisotopes. ${ }^{38}$ The UV-vis spectra of the purified cRGDY-PEG-C' ${ }^{\prime}$ dots exhibited strong absorption at wavelength around $650 \mathrm{~nm}$, corresponding to the absorption maximum of $\mathrm{Cy} 5$ fluorescent dye (Figure 1c). As compared to $\mathrm{C}^{\prime}$ dots without cRGDY surface modification (PEG- $\mathrm{C}^{\prime}$ dots), an additional absorption peak was identified at a wavelength around 275 $\mathrm{nm}$, attributed to the tyrosine residues on the cRGDY peptides (Figure 1c). By dividing the concentrations of Cy5 and cRGDY calculated from the UV-vis spectra by the concentration of $\mathrm{C}^{\prime}$ dots measured by FCS, the numbers of Cy5 and cRGDY per $\mathrm{C}^{\prime}$ dot were estimated to be around 1.6 and 20 , respectively.

For radiolabeling procedures, 4 nmols of purified cRGDY-PEG-C ${ }^{\prime}$ dots were mixed with 1 $\mathrm{mCi}$ of ${ }^{89} \mathrm{Zr}$-oxalate in HEPES buffer $(\mathrm{pH} 8)$ at $75^{\circ} \mathrm{C}$. Radiochemical yields were monitored by radio-TLC. Results showed that, within the first hour, over $50 \%{ }^{89} \mathrm{Zr}$ labeling yield was achieved. A total of $\sim 75 \%{ }^{89} \mathrm{Zr}$ was successfully attached to the particle over a $4 \mathrm{~h}$ radiolabeling period (Figure 2a). As expected, the labeling process was dependent on the particle concentration: the higher the particle-to- ${ }^{89} \mathrm{Zr}$ (nmol-to-mCi) ratio, the higher the ${ }^{89} \mathrm{Zr}$ labeling yield (Figure $2 \mathrm{a}$ ). The specific activity of chelator-free ${ }^{89} \mathrm{Zr}$-labeled cRGDY-PEG-C ${ }^{\prime}$ dots (denoted as cRGDY-PEG- $\left[{ }^{89} \mathrm{Zr}\right] \mathrm{C}^{\prime}$ dots) was found to be in the range of $100-500 \mathrm{Ci} / \mathrm{mmol}$.

Deprotonated silanol groups play a vital role in the chelator-free ${ }^{89} \mathrm{Zr}$ labeling of silica nanoparticles. ${ }^{30}$ When the $\mathrm{pH}$ is below the isoelectric point of silica $(\mathrm{pH} \sim 2-3)$, the surface silanol groups of $\mathrm{C}^{\prime}$ dots will become protonated, making them unsuitable for chelating with positively charged ${ }^{89} \mathrm{Zr}$. This was evidenced by the fact that less than $1 \%$ labeling yield was observed at $\mathrm{pH} 2$ and $75^{\circ} \mathrm{C}$ (Figure 2b). Chelator-free ${ }^{89} \mathrm{Zr}$ labeling was also demonstrated to be temperature-dependent, with higher labeling temperatures leading to faster ${ }^{89} \mathrm{Zr}$ labeling (Figure $2 \mathrm{c}$ ). The optimized labeling $\mathrm{pH}$ and temperature ranges are $\mathrm{pH}$ $8-9$ and $50-75^{\circ} \mathrm{C}$, respectively.

To further demonstrate specific ${ }^{89} \mathrm{Zr}$ labeling of deprotonated silanol groups, remaining silanol groups on the $\mathrm{C}^{\prime}$ dot surface after PEGylation were quenched via the addition of diethoxy dimethyl silane (DEDMS). The resulting modified cRGDY-PEG-C ${ }^{\prime}$ dots were expected to exhibit a lower surface density of reactive silanol groups, thereby reducing the efficiency of chelator-free radiolabeling. ${ }^{39}$ Indeed, an approximate $25 \%$ reduction of ${ }^{89} \mathrm{Zr}$ labeling yield was observed in this case (Figure 2d). Considering that the average specific activity of ${ }^{89} \mathrm{Zr}$-oxalate is about $833 \mathrm{Ci} / \mathrm{mmol}$ of zirconium with a $>99.9 \%$ radiochemical purity, ${ }^{40}$ about $0.14-0.63{ }^{89} \mathrm{Zr}$ per cRGDY-PEG-C ${ }^{\prime}$ dot was estimated for cRGDY-PEG$\left.{ }^{89} \mathrm{Zr}\right] \mathrm{C}^{\prime}$ dots (Table S3). The number of $\mathrm{Zr}$ atoms per particle could be further increased by labeling with cold $\mathrm{Zr}$ (or ${ }^{\text {nat }} \mathrm{Zr}$ ) at varied ratios. As shown in Figure S1, a ${ }^{\text {nat }} \mathrm{Zr}$ density of 
$2.27 \pm 0.08$ could be achieved by labeling cRGDY-PEG-C ${ }^{\prime}$ dots with ${ }^{\text {nat }} \mathrm{Zr}$ at a molar ratio of 1 to 10 . To date, silica-based ${ }^{89} \mathrm{Zr}$ chelator-free radiolabeling has focused exclusively on nanoparticles with a diameter larger than $100 \mathrm{~nm}$ to provide sufficient silanol groups $\left(>10^{5} /\right.$ particle). ${ }^{30,31,41}$ Herein, we show successful ${ }^{89} \mathrm{Zr}$ chelator-free labeling of ultrasmall (6-7 $\mathrm{nm})$ PEGylated silica nanoparticles with a significantly reduced surface and internal silanol group number.

\section{Chelator-Based Zirconium-89 Radiolabeling of cRGDY-PEG-C' Dots}

To achieve traditional chelator-based ${ }^{89} \mathrm{Zr}$ labeling, we used DFO-NCS providing six oxygen donors (Scheme 1b). ${ }^{42}$ In our initial studies, we attached DFO chelator to maleimide functionalized $\mathrm{C}^{\prime}$ dots (mal-cRGDY-PEG-C ${ }^{\prime}$ dots) by introducing glutathione (GSH) as a linker, thereby converting the maleimide groups on $\mathrm{C}^{\prime}$ dot surfaces to primary amine groups for DFO-NCS conjugation. The resulting GSH-modified $\mathrm{C}^{\prime}$ dots were first purified using a PD-10 column and then conjugated with DFO-NCS chelator via the GSH amine groups, resulting in DFO-cRGDY-PEG-C ${ }^{\prime}$ dots for ${ }^{89} \mathrm{Zr}$ labeling. Although high chelator-based labeling yields ( $>80 \%$ ) were achieved after PD-10 purification (to remove free, nonlabeled ${ }^{89} \mathrm{Zr}$ ), very high intestinal uptake of ${ }^{89} \mathrm{Zr}$-DFO-cRGDY-PEG-C ${ }^{\prime}$ dots was observed in a screening PET study (Figure S2a); this finding was hypothesized to be due to the detachment of ${ }^{89} \mathrm{Zr}$-DFO-GSH from the particles. No obvious bone uptake was observed at $24 \mathrm{~h}$ postinjection, indicating no detachment of free ${ }^{89} \mathrm{Zr}$ from the radio-conjugates (Figure S2a).

To solve this problem, primary amine groups were attached directly to the $\mathrm{C}^{\prime}$ dot surface using a recently developed post-PEGylation surface modification by insertion (PPSMI) method. ${ }^{43}$ To that end, after $\mathrm{C}^{\prime}$ dot PEGylation, additional amino-silane molecules were added to the reaction and inserted into the PEG layer, attaching to the silica surface underneath. The resulting $\mathrm{NH}_{2}$-cRGDY-PEG- $\mathrm{C}^{\prime}$ dots contained reactive amine groups on the silica surface under the PEG layer, allowing for further conjugation with e.g., NCS functionalized DFO chelators. After purification, the $\mathrm{NH}_{2}$-cRGDY-PEG-C ${ }^{\prime}$ dots exhibited good product quality, similar to that of cRGDY-PEG-C ${ }^{\prime}$ dots without amine functionalization (Figures $1 \mathrm{~d}-\mathrm{f}$ ). The average diameter of the purified $\mathrm{NH}_{2}$-cRGDY-PEG-C dots was around $6.5 \mathrm{~nm}$. The numbers of $\mathrm{Cy} 5$ and cRGDY peptides per $\mathrm{C}^{\prime}$ dot were estimated to be around 1.5 and 18 , respectively (Figures $1 \mathrm{~d}-\mathrm{f}$ ). The purified $\mathrm{NH}_{2}$-cRGDYPEG-C ${ }^{\prime}$ dots were then conjugated with DFO-NCS using a reaction molar ratio of 1:20 between the particles and DFO-NCS, followed by purification using a PD-10 column to remove unreacted DFO-NCS. Labeling of ${ }^{89} \mathrm{Zr}$-oxalate to the resulting DFO-cRGDY-PEG-C ' dots was performed at $37^{\circ} \mathrm{C}$ for $60 \mathrm{~min}$. A nearly $100 \%$ labeling yield was achieved by using a particle-to- ${ }^{89} \mathrm{Zr}$ ratio of $0.4 \mathrm{nmol} / 1 \mathrm{mCi}$ (Figure $2 \mathrm{e}$ ). The specific activity was estimated to be in the range of $1300-4300 \mathrm{Ci} / \mathrm{mmol}$, significantly higher than results from the chelator-free method. About $1.59-5.14{ }^{89} \mathrm{Zr}$ per $\mathrm{C}^{\prime}$ dot was estimated in the final ${ }^{89} \mathrm{Zr}$ DFO-cRGDY-PEG-C ${ }^{\prime}$ dot product (Table S4). To estimate the number of accessible DFO per particle, as-synthesized DFO-cRGDY-PEG-C ${ }^{\prime}$ dots were first labeled with ${ }^{\text {nat }} \mathrm{Zr}$ and then subjected to ${ }^{\text {nat }} \mathrm{Zr}$ quantification using microwave plasma-atomic emission spectroscopy (MP-AES). Our results revealed an average of $3.42 \pm 0.13^{\text {nat }} \mathrm{Zr}$ per $\mathrm{C}^{\prime}$ dot for ${ }^{\text {nat }} \mathrm{Zr}$-DFOcRGDY-PEG-C ${ }^{\prime}$ dots synthesized at a particle to DFO ratio of $1: 10$ and $4.76 \pm 0.13$ for a 
1:30 ratio (Figure $2 \mathrm{f}$ ). Because excess ${ }^{\text {nat }} \mathrm{Zr}$ was used during the labeling and unreacted ${ }^{\text {nat }} \mathrm{Zr}$ was removed by chelating with EDTA, the number of nat $\mathrm{Zr}$ per $\mathrm{C}^{\prime}$ dot (about 3-5) should be a good measure of the number of accessible DFO per DFO-cRGDY-PEG-C ${ }^{\prime}$ dot. A subsequent pilot PET study showed a significantly reduced intestinal uptake using aminebased ${ }^{89} \mathrm{Zr}$-DFO-cRGDY-PEG-C' ${ }^{\prime}$ dots (Figure S2b).

\section{Radiostability and Blood Circulation Half-Lives of ${ }^{89}$ Zr-Labeled cRGDY-PEG-C' Dots}

We next investigated the in vitro and in vivo radiostability and blood circulation half-lives of the chelator-free and chelator-based ${ }^{89}$ Zr-labeled cRGDY-PEG-C' ${ }^{\prime}$ dot probes. High radiostability is vital because PET detects the radioisotope itself rather than the particles. Both ${ }^{89} \mathrm{Zr}$-labeled cRGDY-PEG-C' ${ }^{\prime}$ dot probes were synthesized and purified using PD-10 columns. Figure $\mathrm{S} 3$ shows representative elution profiles of chelator-free and chelatorbased ${ }^{89} \mathrm{Zr}$-labeled cRGDY-PEG-C' dot probes in PD-10 columns. The fraction from 2.5 to $4.0 \mathrm{~mL}$ was collected for subsequent studies.

The radiostabilities of both ${ }^{89} \mathrm{Zr}$-labeled cRGDY-PEG-C ${ }^{\prime}$ dot probes were found to be comparable in phosphate-buffered saline (PBS) over a $120 \mathrm{~h}$ time interval at $37{ }^{\circ} \mathrm{C}$ under stirring at $650 \mathrm{rpm}$. Both ${ }^{89} \mathrm{Zr}$-DFO-cRGDY-PEG-C ${ }^{\prime}$ dots and cRGDY-PEG- $\left[{ }^{89} \mathrm{Zr}\right] \mathrm{C}^{\prime}$ dots also demonstrated greater than $99 \%$ radiopurity in PBS (Figure 3a). Similar results were found when incubating both ${ }^{89} \mathrm{Zr}$-labeled cRGDY-PEG-C ${ }^{\prime}$ dot probes in human serum under the same conditions (Figure $3 \mathrm{~b}$ ). To evaluate in vivo radiostability of ${ }^{89} \mathrm{Zr}$-labeled probes, non tumor-bearing nude mice were intravenously (i.v.)-injected with $\sim 200 \mu \mathrm{Ci}$ ( 7.4 $\mathrm{MBq}$ ) of ${ }^{89} \mathrm{Zr}$-labeled cRGDY-PEG-C' ${ }^{\prime}$ dots. Whole blood specimens were collected at 2 , 24 , and $48 \mathrm{~h}$ postinjection, and the plasma fraction (which contained $>98 \%$ of the ${ }^{89} \mathrm{Zr}$ labeled cRGDY-PEG-C ${ }^{\prime}$ dots) was separated from the whole blood at different postinjection time points by centrifugation at $8000 \mathrm{rpm}$ for $10 \mathrm{~min}$ and used to test radiopurity. The nonspecific association of ${ }^{89} \mathrm{Zr}$-labeled cRGDY-PEG-C ${ }^{\prime}$ dots with red blood cells was estimated to be less than $2 \%$. The percentage of intact ${ }^{89} \mathrm{Zr}$-labeled cRGDY-PEG-C ${ }^{\prime}$ dots was also measured by radio-TLC. As shown in Figure 3c, $>98 \%$ of intact ${ }^{89} \mathrm{Zr}$-DFOcRGDY-PEG-C ${ }^{\prime}$ dots was estimated at $48 \mathrm{~h}$ postinjection in mouse plasma, while $<75 \%$ was found for mice injected with cRGDY-PEG- $\left.{ }^{89} \mathrm{Zr}\right] \mathrm{C}^{\prime}$ dots. The latter finding suggests detachment of free ${ }^{89} \mathrm{Zr}$ during its circulation in vivo and highlights the dramatic difference in product radiostability found under in vitro and in vivo conditions.

To evaluate the circulation half-life, blood from mice i.v.-injected with ${ }^{89} \mathrm{Zr}$-labeled cRGDYPEG- $C^{\prime}$ dots was sampled at various postinjection time points and assayed by gamma counting $(n=3)$. Blood uptake values were converted to a percentage of the injected dose per gram (\% ID/g), and fit with a two-compartment model. As shown in Figures $3 \mathrm{~d}$ and e, nearly equivalent blood circulation half-lives of about $15 \mathrm{~h}$ were measured, greater than those previously published for earlier generation radioiodinated particles (Table S1). ${ }^{16,17}$

\section{Dynamic PET Imaging Using ${ }^{89}$ Zr-Labeled cRGDY-PEG-C' Dots}

PET is a widely used molecular imaging modality for noninvasively and quantitatively tracking the pharmacokinetics (PK) of various types of radiolabeled probes in vivo with high sensitivity. ${ }^{44}$ Limited by tissue penetration depths, it is well-known that optical imaging is 
generally not suitable for in vivo whole body screening and quantification of particle distributions within tissues. To track the distribution and fast renal clearance of systemically injected $\mathrm{C}^{\prime}$ dots, particularly in the early postinjection time period, a 60 min dynamic PET imaging study was performed in representative mice, each animal injected with one of the two ${ }^{89} \mathrm{Zr}$-labeled cRGDY-PEG-C ${ }^{\prime}$ dot probes. As shown in Figures $4 \mathrm{a}$ and $\mathrm{b}$, maximum intensity projection (MIP) images show marked activity of ${ }^{89} \mathrm{Zr}$-labeled cRGDY-PEG-C' dots in the mouse heart immediately after i.v. injection. Gradually reduced heart activity was observed in both cases with overall activity concentration estimated to be $20.5 \% \mathrm{ID} / \mathrm{g}$ at 60 min postinjection for mice injected with cRGDY-PEG- $\left[{ }^{89} \mathrm{Zr}\right] \mathrm{C}^{\prime}$ dots (Figure $4 \mathrm{c}$ ) and $19.3 \%$ ID/g for mice injected with ${ }^{89} \mathrm{Zr}$-DFO-cRGDY-PEG-C ${ }^{\prime}$ dots (Figure 4d). A similar trend was observed for hepatic uptake with $60 \mathrm{~min}$ postinjection uptake values of both probes estimated to be $\sim 6.5 \% \mathrm{ID} / \mathrm{g}$. Significant kidney and bladder uptake was observed as early as 5 min postinjection, observed in both the MIP images and time-activity curves, clearly highlighting renal clearance capabilities of both ${ }^{89} \mathrm{Zr}$-labeled cRGDY-PEG-C ${ }^{\prime}$ dot probes. Short videos of the first $1 \mathrm{~h}$ dynamic biodistribution patterns of mice i.v.-injected with ${ }^{89} \mathrm{Zr}$ labeled cRGDY-PEG-C' ${ }^{\prime}$ dot probes were also provided as Videos S1 and S2.

\section{In Vivo Pharmacokinetics and Radiation Dosimetry Studies}

Detailed biodistribution studies were performed to investigate the uptake of both ${ }^{89} \mathrm{Zr}$ labeled cRGDY-PEG-C ${ }^{\prime}$ dot probes at various postinjection time points after harvesting, weighing, and assaying major organs/tissues of interest from mice at the termination of the study (i.e., 5, 24, and 72 h, Tables S5 and S6, Figure 5). As evidenced in the dynamic PET imaging studies (Figure 4), the biodistribution studies confirmed significant activity of both ${ }^{89} \mathrm{Zr}$-labeled cRGDY-PEG-C ${ }^{\prime}$ dot probes in the blood compartment (Figures 5a and b). Plasma activity concentrations were twice as high as those for whole blood (Figure S4). Not surprisingly, urine activity at early postinjection time points varied from mouse to mouse, ranging from $<10 \% \mathrm{ID} / \mathrm{g}$ to $>20 \% \mathrm{ID} / \mathrm{g}$. A total of $60-70 \%$ ID of ${ }^{89} \mathrm{Zr}$-labeled cRGDY-PEG$\mathrm{C}^{\prime}$ dot probes was cleared within $72 \mathrm{~h}$ postinjection in the current study. As opposed to representative findings for $>10 \mathrm{~nm}$ sized nanoparticles, usually revealing marked hepatic uptake (i.e., 30-99\% ID), ${ }^{6}$ both ${ }^{89} \mathrm{Zr}$-labeled cRGDY-PEG-C ${ }^{\prime}$ dot probes exhibited significantly lower hepatic uptake ( $<5 \%$ ID/g). Interestingly, ${ }^{89} \mathrm{Zr}$-labeled cRGDY-PEG-C ${ }^{\prime}$ dots also showed only $2-4 \% \mathrm{ID} / \mathrm{g}$ in the kidney, which was significantly lower (about 5-10 fold less) than what has previously been described for other renally clearable ultrasmall particles such as ultrasmall quantum dots ${ }^{9}$ or gold nanoparticles. ${ }^{11,12}$ This suggests that in addition to size, optimized surface chemical particle properties are important for low offtarget particle accumulation.

A noticeable difference in overall bone uptake was found between the two ${ }^{89} \mathrm{Zr}$-labeled cRGDY-PEG-C ${ }^{\prime}$ dot probes. Values started to increase beyond 5 and $10 \% \mathrm{ID} / \mathrm{g}$ at the 24 and $72 \mathrm{~h}$ post i.v.-injection time points, respectively, for cRGDY-PEG- $\left.{ }^{89} \mathrm{Zr}\right] \mathrm{C}^{\prime}$ dots (Figure $5 \mathrm{c}$, $p<0.005)$. Such high bone uptake likely does not reflect marrow accumulation of cRGDYPEG- $\left[{ }^{89} \mathrm{Zr}\right] \mathrm{C}^{\prime}$ dot probes but rather indicates ongoing detachment of the free ${ }^{89} \mathrm{Zr}^{4+}$ from the cRGDY-PEG- $\left[{ }^{89} \mathrm{Zr}\right] \mathrm{C}^{\prime}$ dots due to relatively low radiostability in vivo (Figure $3 b$ ). Free ${ }^{89} \mathrm{Zr}^{4+}$ is an osteophilic cation which could be readily accreted into bone mineral, ${ }^{45}$ as shown in Figure S5. Monitoring the change in bone uptake over time has also been 
demonstrated as one of the best ways to study the in vivo stability of ${ }^{89} \mathrm{Zr}$-labeled nanoprobes. ${ }^{30}$ Attempts to reduce the bone uptake of cRGDY-PEG- $\left[{ }^{89} \mathrm{Zr}\right] \mathrm{C}^{\prime}$ dots by removing the less well-chelated surface ${ }^{89} \mathrm{Zr}$ from cRGDY-PEG- $\left[{ }^{89} \mathrm{Zr}\right] \mathrm{C}^{\prime}$ dots using EDTA challenge prior to injection was demonstrated to be only marginally effective in minimizing bone uptake (Figure S6). Only 20\% bone uptake reduction was observed even after overnight EDTA challenge (conditions: $10 \mathrm{mM}$ EDTA, $37{ }^{\circ} \mathrm{C}$, kept under stirring at 650 rpm, Figure S6c). PET imaging in Figure S6b reveals obvious and persistent bone and joint uptake of cRGDY-PEG-[ $\left.{ }^{89} \mathrm{Zr}\right] \mathrm{C}^{\prime}$ dots that were subjected to an additional EDTA challenge process. As reported previously, ${ }^{45}$ vthe clearance of ${ }^{89} \mathrm{Zr}$ from the bones of mice was found to be slow with no significant reduction after 1 week (Figure S7). The excess and retained accumulation of radioactive ${ }^{89} \mathrm{Zr}^{4+}$ in the bone marrow can increase the radiation dose to this compartment (an especially radiosensitive tissue), potentially hindering clinical translation.

To estimate mean organ absorbed doses and the effective dose in a 70-kg standard man, dosimetry calculations for both ${ }^{89} \mathrm{Zr}$-labeled cRGDY-PEG-C' dot probes were performed based on the biodistribution data shown in Figure 5 and using the OLINDA computer program (yielding doses expressed in $\mathrm{mSv} / \mathrm{MBq}{ }^{89} \mathrm{Zr}$ administered). ${ }^{46}$ Table $\mathrm{S} 7$ compares the estimated tissue-absorbed dose in humans for both ${ }^{89} \mathrm{Zr}$-labeled cRGDY-PEG-C ${ }^{\prime}$ dot probes. A slightly higher absorbed dose $(0.084 \mathrm{mSv} / \mathrm{MBq})$ in red marrow was found for the chelator-free ${ }^{89} \mathrm{Zr}$-labeled cRGDY-PEG- $\left[{ }^{89} \mathrm{Zr}\right] \mathrm{C}^{\prime}$ dot probe when compared to the chelatorbased ${ }^{89} \mathrm{Zr}$-labeled ${ }^{89} \mathrm{Zr}$-DFO-cRGDY-PEG-C ${ }^{\prime}$ dot probe $(0.062 \mathrm{mSv} / \mathrm{MBq})$. An absorbed dose $\sim 0.1 \mathrm{mSv} / \mathrm{MBq}$ was estimated for both ${ }^{89} \mathrm{Zr}$-labeled cRGDY-PEG-C ${ }^{\prime}$ dot probes in the human liver, only one-tenth of a previously reported value for ${ }^{89} \mathrm{Zr}$-DFO-trastuzumab (liver uptake was $\sim 12 \% \mathrm{ID}$, average estimated absorbed dose in liver was $1.54 \mathrm{mSv} / \mathrm{MBq}){ }^{47}$ Although significantly higher bone uptake was observed in the small-animal study, the estimated radiation dosimetry in a 70-kg standard man showed only a minor increase $(<20 \%)$ in both the total-body and effective dose for the chelator-free ${ }^{89} \mathrm{Zr}$-labeled cRGDYPEG- $\left[{ }^{89} \mathrm{Zr}\right] \mathrm{C}^{\prime}$ dot product. Taken together, in vivo pharmacokinetic studies confirmed the renal clearance and extended blood circulation of ${ }^{89} \mathrm{Zr}$-labeled cRGDY-PEG-C ${ }^{\prime}$ dot probes within the first $24 \mathrm{~h}$ postinjection. All major organs, especially liver, spleen and kidney, showed minor $(<5 \% \mathrm{ID} / \mathrm{g}$ ) uptake throughout the study period. The only major difference between the chelator-free and the chelator-based ${ }^{89} \mathrm{Zr}$-labeled cRGDY-PEG-C ${ }^{\prime}$ dot probes is the lower in vivo radiostability and significantly ( $2-4$ fold) higher bone uptake of the former at $24 \mathrm{~h}$ postinjection. However, the radiation dosimetry analysis showed favorable total-body and effective doses for both ${ }^{89} \mathrm{Zr}$-labeled cRGDY-PEG-C ${ }^{\prime}$ dot probes, which encouraged us to explore in vivo tumor-specific targeting of both radio-labeled nanoprobes in wellcharacterized integrin $a_{\mathrm{v}} \beta_{3}$-expressing human melanoma xenograft models.

\section{In Vivo Tumor-Targeting by PET Imaging}

Designing a "target-or-clear" multifunctional nanoparticle platform that can specifically localize in the target of interest after systemic administration while maintaining low nonspecific accumulations in the RES has long been one of the major challenges in the field of nanomedicine. Table S1 lists the current research status of ultrasmall nanoparticles exhibiting both renal clearance and in vivo tumor-targeting capabilities. Following 
systematic investigations and comparisons of ${ }^{89} \mathrm{Zr}$ labeling strategies, we turned our attention to studying in vivo tumor-specific targeting and renal clearance profiles for both ${ }^{89} \mathrm{Zr}$-labeled cRGDY-PEG-C ${ }^{\prime}$ dot probes in $a_{\mathrm{v}} \beta_{3}$ integrin-expressing melanoma xenograft models.

As shown in Figure 6, significant bladder activity was observed in the $2 \mathrm{~h}$ MIP images for mice injected with cRGDY-PEG- $\left.{ }^{89} \mathrm{Zr}\right] \mathrm{C}^{\prime}$ dots (Figure 6a) and ${ }^{89} \mathrm{Zr}$-DFO-cRGDY-PEG-C ${ }^{\prime}$ dots (Figures $6 \mathrm{~b}$ and $\mathrm{c}$ ). The high cardiac uptake observed ( $\sim 20 \% \mathrm{ID} / \mathrm{g}$ ) clearly indicated the circulation of ${ }^{89} \mathrm{Zr}$-labeled cRGDY-PEG-C ${ }^{\prime}$ dots in the blood compartment. Time-activity curves shown in Figures 6d-f show the clearance of ${ }^{89} \mathrm{Zr}$-labeled cRGDY-PEG-C ${ }^{\prime}$ dots from the blood with uptake values estimated to be about 5-6 and 1-2\% ID/g at 24 and $72 \mathrm{~h}$ postinjection, respectively. The clearance of ${ }^{89} \mathrm{Zr}$-labeled cRGDY-PEG-C ${ }^{\prime}$ dots by the RES (e.g., liver) was estimated to be only 5-6\% ID/g at $2 \mathrm{~h}$ postinjection with slight reductions down to $4-5 \% \mathrm{ID} / \mathrm{g}$ after 3 days; these values are markedly lower than previously reported values for particles larger than $10 \mathrm{~nm}^{6}$ and close to that of early generation ${ }^{124}$ I-labeled C dots having a similar HD size (liver uptake: $2-4 \% \mathrm{ID} / \mathrm{g}$ ). ${ }^{16}$ Splenic uptake was found to be only half of that found for liver uptake over the course of three days. Muscle uptake was found to be as low as $\sim 1 \% \mathrm{ID} / \mathrm{g}$. Given findings compatible with bulk renal clearance, significantly reduced RES uptake, and very low background activity levels in muscle, together with blood circulation half-life of $\sim 15 \mathrm{~h}$, significantly enhanced tumor-tobackground ratios may therefore be achievable.

As shown in Figures $6 \mathrm{a}$ and $\mathrm{b}$, high M21 ( $a_{\mathrm{v}} \beta_{3}$-positive) tumor uptake was observed in mice injected with both cRGDY-PEG-[ $\left.{ }^{89} \mathrm{Zr}\right] \mathrm{C}^{\prime}$ dots (Figure 6a, $10.1 \pm 2.1 \% \mathrm{ID} / \mathrm{g}$ ) and ${ }^{89} \mathrm{Zr}$ DFO-cRGDY-PEG-C ${ }^{\prime}$ dots (Figure $6 \mathrm{~b}, 10.5 \pm 4.0 \% \mathrm{ID} / \mathrm{g}$ ) at $2 \mathrm{~h}$ postinjection. The tumor uptake peaked at $24 \mathrm{~h}$ postinjection with an additional slight increase to about $10.7 \pm 1.3$ and $12.0 \pm 1.4 \% \mathrm{ID} / \mathrm{g}$, respectively (Figure $6 \mathrm{~g}$ ). Over 5 -fold enhancement of tumor uptake was estimated when compared with our first-generation C dots (cRGDY ligand density: 6) labeled with ${ }^{124}$ ( (maximal M21 tumor uptake: 2\% ID/g at $4 \mathrm{~h}$ postinjection). ${ }^{16}$ Retention of particle activity over the $72 \mathrm{~h}$ time period was observed in M21 tumor-bearing mice injected with both types of ${ }^{89} \mathrm{Zr}$-labeled cRGDY-PEG-C ${ }^{\prime}$ dot probes (Figures $6 \mathrm{~d}$ and e), noting only a very slow wash-out rate. As expected, mice injected with the chelatorfree ${ }^{89} \mathrm{Zr}$-labeled cRGDY-PEG-C ${ }^{\prime}$ dots showed detachment of free ${ }^{89} \mathrm{Zr}$, with its accumulation in bone, joint, and spine (Figures $6 \mathrm{a}$ and $S 8$ ), while significantly reduced bone and joint uptake was found in mice injected with ${ }^{89} \mathrm{Zr}$-DFO-cRGDY-PEG-C ${ }^{\prime}$ dots (Figures $6 \mathrm{~b}$ and S8). Maximizing target tissue uptake and retention as well as target-to-background ratios in preclinical models are key considerations for the rational design of targeted nanoprobes for clinical cancer care. ${ }^{5}$ Recent debates in the field of nanomedicine have focused on nanoparticle delivery efficiency, the extent to which enhanced permeability and retention effects promote target accumulation, nanoparticle-tumor interactions, and nonspecific uptake at off-target sites, among others, highlighting the significant challenges encountered in the quest to translate nanoprobes to the clinic. ${ }^{48-53}$ Other areas of active investigation critical to the successful navigation of these challenges require the integration of high-resolution analytical approaches to improve particle characterization, facilitate particle clearance, and enhance particle penetration and diffusion within tumors. 
A control study was performed in M21-L tumor-bearing mice ( $a_{\mathrm{v}} \beta_{3}$-negative) following injection of ${ }^{89} \mathrm{Zr}$-DFO-cRGDY-PEG-C ${ }^{\prime}$ dots to further demonstrate target specificity of ${ }^{89} \mathrm{Zr}$-labeled cRGDY-PEG- $\mathrm{C}^{\prime}$ dots. Findings showed similar particle distributions in major organs/tissues such as bladder, heart, liver, and muscle with significantly lower uptake in the M21-L tumors (on average 2-3\% ID/g), as shown in Figures 6c, 6f, and S9. For mice injected with either cRGDY-PEG- $\left[{ }^{89} \mathrm{Zr}\right] \mathrm{C}^{\prime}$ dots or ${ }^{89} \mathrm{Zr}$-DFO-cRGDY-PEG-C ${ }^{\prime}$ dots, tumor accumulation rates and tumor-to-background ratios demonstrated no statistically significant differences (Figures 6g-j and S9). For mice injected with ${ }^{89} \mathrm{Zr}$-DFO-cRGDY-PEG-C ${ }^{\prime}$ dots, maximum tumor-to-muscle ratios were found to be $9.6 \pm 2.5$ at $72 \mathrm{~h} \mathrm{p}$.i., which is 3 -fold higher than that measured in M21-L tumor-bearing mice (i.e., $2.8 \pm 0.7$, Figure 6j) and 2fold higher than that found for ${ }^{124}$ I-labeled $\mathrm{C}$ dots (maximal tumor-to-muscle: $~ 5$ ). ${ }^{16}$ Finally, on the basis of high tumor uptake and low RES accumulation, we observed tumorto-liver ratios of about 2 or higher in M21 tumor-bearing mice injected with cRGDY-PEG$\left[{ }^{89} \mathrm{Zr}\right] \mathrm{C}^{\prime}$ dots or ${ }^{89} \mathrm{Zr}$-DFO-cRGDY-PEG-C ${ }^{\prime}$ dots (Figure 6i), one of the unique features distinguishing ${ }^{89} \mathrm{Zr}$-labeled cRGDY-PEG-C' ${ }^{\prime}$ dot probes from other tumor-targeting particles. ${ }^{6}$ Taken together, we successfully demonstrated in vivo targeting specificity and concomitant renal clearance for both ${ }^{89} \mathrm{Zr}$-labeled cRGDY-PEG-C' ${ }^{\prime}$ dot preparations in $a_{\mathrm{v}} \beta_{3}$ integrin-expressing melanoma xenograft models.

\section{CONCLUSIONS}

In conclusion, to address challenges in the radiolabeling of ultrasmall renally clearable cRGDY-PEG-C' ${ }^{\prime}$ dots, we developed and compared two ${ }^{89} \mathrm{Zr}$-radiolabeling strategies based on their biological and dosimetric properties. Although comparable in vitro radiostability was found for both radioconjugates, chelator-based radiolabeling showed a significantly higher in vivo radiostability than chelator-free preparations. Both PK studies and PET imaging evaluations confirmed renal clearance, low RES accumulation, enhanced tumor uptake, and high target-to-background ratios for both products in $a_{\mathrm{v}} \beta_{3}$ integrin-expressing human melanoma xenograft models. While there are limitations to rodent tumor models, they nonetheless provide useful information that contributes to the clinical translation of nanoparticles and other agents. In particular, the findings presented herein point to the favorable translatability of these novel target-or-clear ${ }^{89} \mathrm{Zr}$-labeled cRGDY-PEG-C ${ }^{\prime}$ dot tracers to human subjects for systemic targeted detection of cancer.

\section{EXPERIMENTAL SECTION}

\section{Synthesis, Purification, and Characterization of cRGDY-PEG-C' Dots and Amine- Functionalized $\mathrm{NH}_{2}$-cRGDY-PEG-C' Dots}

cRGDY-PEG-C ${ }^{\prime}$ dots and $\mathrm{NH}_{2}$-cRGDY-PEG-C $\mathrm{C}^{\prime}$ dots were synthe-sized as described previously, ${ }^{21,43}$ the latter using a recently reported post-PEGylation surface modification by insertion approach. More specifically, a typical synthesis of cRGDY-PEG-C ${ }^{\prime}$ dots started with dissolving $5.7 \mu \mathrm{mol}$ of NHS ester/maleimido functionalized heterofunctional polyethylene glycol (PEG) with molar mass around 866, referred to as mal-PEG-NHS, in $24.8 \mu \mathrm{L}$ of dimethyl sulfoxide (DMSO). The solution was mixed with $5.2 \mu \mathrm{mol}$ of (3aminopropyl)-triethoxysilane (amine-silane) at room temperature under nitrogen. The 
reaction mixture was kept at room temperature under nitrogen for two days to complete conjugation of mal-PEG-NHS with amine-silane, forming mal-PEG-silane. Afterward, 6.3 $\mu \mathrm{mol}$ of cyclo(Arg-Gly-Asp-D-Tyr-Cys) peptide (cRGDY), dissolved in $300 \mu \mathrm{L}$ DMSO, was further added into the mixture at room temperature under nitrogen. The reaction mixture was then kept at room temperature under nitrogen overnight to complete conjugation of malPEG-silane with cRGDY peptide, forming cRGDY-PEG-silane. At the same time, $0.5 \mu \mathrm{mol}$ of maleimido functionalized Cy5 dye (Cy5-mal), dissolved in $30 \mu \mathrm{L}$ DMSO, was mixed with $9.5 \mu \mathrm{mol}$ of (3-mercaptopropyl)-trimethoxysilane (thiol-silane). The mixture was kept at room temperature under nitrogen overnight to complete conjugation of Cy5-mal with thiol-silane, forming Cy5-silane.

In the next step, $68 \mu \mathrm{L}$ of tetramethyl orthosilicate (TMOS liquid) and conjugated Cy5silane were added into $10 \mathrm{~mL}$ of aqueous solution of ammonium hydroxide at $\mathrm{pH}$ around 8.5. The addition was conducted at room temperature under vigorous stirring $(600 \mathrm{rpm})$. The reaction solution was then kept at room temperature under vigorous stirring overnight. Afterward, cRGDY-PEG-silane prepared in the previous step was added into the reaction mixture at room temperature under vigorous stirring, followed by the addition of $100 \mu \mathrm{L}$ of silane functionalized PEGs (PEG-silane liquid) with molar mass around 500. Afterward, the reaction solution was kept at room temperature overnight under vigorous stirring. The reaction solution was then kept at $80^{\circ} \mathrm{C}$ without stirring overnight to further enhance the covalent attachment of PEG-silane and cRGDY-PEG-silane to silica nanoparticle surface. After cooling the reaction solution to room temperature, the resulting cRGDY-PEG-Cy5-C ${ }^{\prime}$ dots were purified by GPC, filtered by sterile syringe filters, and finally stored at $4{ }^{\circ} \mathrm{C}$. Where applicable, remaining silanol groups on cRGDY-PEG-C $\mathrm{C}^{\prime}$ dots could be terminated by adding diethoxydimethylsilane (DEDMS) to the synthesis mixture at a concentration of $7.3 \mathrm{mM}$ after the reaction temperature was reduced from $80^{\circ} \mathrm{C}$ to room temperature. The reaction solution was then kept at room temperature under vigorous stirring overnight before purification, allowing DEDMS to covalently attach to the remaining silanol groups on the silica particle surface. The rest of the synthesis remained the same as that of the regular cRGDY-PEG-C' ${ }^{\prime}$ dots. $^{21}$

The synthesis of $\mathrm{NH}_{2}$-cRGDY-PEG-C ${ }^{\prime}$ dots followed the same protocol as that for cRGDYPEG-C ${ }^{\prime}$ dots, except that additional amine-silane was inserted into the PEG layer of cRGDY-PEG-C ${ }^{\prime}$ dots and attached to the silanol groups underneath, as described in detail in ref 43. More specifically, after the reaction temperature was reduced from $80^{\circ} \mathrm{C}$ to room temperature in the synthesis of cRGDY-PEG-C ${ }^{\prime}$ dots, $8.6 \mu \mathrm{mol}$ of amine-silane was added into the reaction solution at room temperature under vigorous stirring. The reaction was kept at room temperature overnight under vigorous stirring before particle purification to complete amine functionalization. ${ }^{43}$

Purification and characterization methods for different $C^{\prime}$ dots, including GPC purification as well as TEM, FCS, and UV-vis measurements, are detailed in our previous publications. ${ }^{21,43}$ In short, GPC purification and characterization were performed using a BioLogic LP system equipped with a $275 \mathrm{~nm}$ UV detector. The column used was an EconoColumn chromatography column packed with Superdex 200 resin from GE healthcare. TEM images were taken on a FEI Tecnai T12 Spirit TEM operated at an acceleration voltage of 
$120 \mathrm{kV}$. FCS measurements were conducted using a home-built FCS setup with a $633 \mathrm{~nm}$ solid state laser as the excitation source. FCS error bars were obtained from a combination of the standard deviation from multiple measurements and a systemic error resulting from FCS alignment variations. UV-vis measurements were performed using a Varian Cary 5000 spectrophotometer.

\section{${ }^{89} \mathrm{Zr}$-Oxalate Production}

${ }^{89} \mathrm{Zr}$ was produced at Memorial Sloan Kettering Cancer Center on a TR19/9 cyclotron (Ebco Industries Inc.) via the ${ }^{89} \mathrm{Y}(\mathrm{p}, \mathrm{n})^{89} \mathrm{Zr}$ reaction and purified to yield ${ }^{89} \mathrm{Zr}$ with a specific activity of $5.28-13.43 \mathrm{mCi} / \mu \mathrm{g}(470-1195 \mathrm{Ci} / \mathrm{mmol})$ of zirconium. ${ }^{40}$ Activity measurements were performed using a CRC-15R Dose Calibrator (Capintec). For the quantification of activities, experimental samples were counted on an Automatic Wizard ${ }^{2} \gamma$-Counter (PerkinElmer). All in vivo experiments were performed according to protocols approved by the Memorial Sloan Kettering Institutional Animal Care and Use Committee. A purity of greater than $95 \%$ was confirmed using radio-TLC for all of the ${ }^{89} \mathrm{Zr}$-labeled cRGDY-PEG-C ' dots.

\section{Chelator-Free ${ }^{89} \mathrm{Zr}$ Radiolabeling of c(RDGyC)-PEG-C' Dots}

For a typical chelator-free ${ }^{89} \mathrm{Zr}$ labeling of cRGDY-PEG-C' ${ }^{\prime}$ dots, 4 nmol of cRGDY-PEG-C ' dots was mixed with $1 \mathrm{mCi}$ of ${ }^{89} \mathrm{Zr}$-oxalate in HEPES buffer (pH 8) at $75^{\circ} \mathrm{C}$. The radiolabeling yield of cRGDY-PEG- $\mathrm{C}^{\prime}$ dots at $1,30,60,120$, and 240 min was monitored using salicylic acid impregnated instant thin-layer chromatography paper (ITLCSA) (Agilent Technologies), and analyzed either on a Bioscan AR-2000 radio-TLC plate reader using Winscan Radio-TLC software (Bioscan Inc., Washington, DC) or an Automatic Wizard $^{2} \gamma$-Counter (PerkinElmer). After incubation, $5 \mu \mathrm{L}$ aliquots were withdrawn and mixed with $50 \mu \mathrm{L}$ of EDTA ( $50 \mathrm{mM}$, pH 5-6) before analyzing by ITLC using EDTA (50 $\mathrm{mM}, \mathrm{pH} 5-6)$ as a mobile phase solvent. Free ${ }^{89} \mathrm{Zr}$ formed an instantaneous complex with EDTA and eluted with the solvent front, while ${ }^{89} \mathrm{Zr}$-labeled cRGDY-PEG-C ${ }^{\prime}$ dots remained at the origin. For more accurate quantification, strips were cut in half, and $\gamma$-ray emissions at $909 \mathrm{keV}$ were counted on a calibrated $\boldsymbol{\gamma}$-counter (PerkinElmer) using a dynamic energy window of 800-1000 keV. Similar procedures were introduced when studying the $\mathrm{pH}-$, concentration-, and temperature-dependent chelator-free labeling of cRGDY-PEG- $\mathrm{C}^{\prime}$ dots. The specific activity of chelator-free ${ }^{89} \mathrm{Zr}$-labeled cRGDY-PEG-C' ${ }^{\prime}$ dots was found to be in the range of $100-500 \mathrm{Ci} / \mathrm{mmol}$.

\section{Synthesis and Chelator-Based ${ }^{89} \mathrm{Zr}$ Labeling of DFO-cRGDY-PEG-C' Dots}

A traditional chelator-based ${ }^{89} \mathrm{Zr}$ labeling technique was introduced by reacting $\mathrm{NH}_{2}$ cRGDY-PEG-C' ${ }^{\prime}$ dots with DFO-NCS (molar ratio was 1:20) for $1-2 \mathrm{~h}$ at $22{ }^{\circ} \mathrm{C}$, $\mathrm{pH} 8-9$, and kept under stirring at $640 \mathrm{rpm}$. As synthesized DFO-cRGDY-PEG- $\mathrm{C}^{\prime}$ dots were then purified by passing the particles through a PD-10 column using phosphate-buffered saline (PBS) as the mobile phase. For chelator-based ${ }^{89} \mathrm{Zr}$ labeling, $0.2-0.75 \mathrm{nmol}$ of DFOcRGDY-PEG-C ${ }^{\prime}$ dots were then mixed with $1 \mathrm{mCi}$ of ${ }^{89} \mathrm{Zr}$-oxalate in HEPES buffer (pH 8) at $37^{\circ} \mathrm{C}$ for 1,30 , and $60 \mathrm{~min}$; final labeling $\mathrm{pH}$ was kept as 7-7.5. The labeling yield was monitored as described above. An EDTA challenge process was introduced to remove any nonspecifically bound ${ }^{89} \mathrm{Zr}$. As synthesized ${ }^{89} \mathrm{Zr}$-DFO-cRGDY-PEG-C' dots were then 
purified by using a PD-10 column. The final radiochemical purity was measured using ITLC. The specific activity was found to be in the range of $1300-4300 \mathrm{Ci} / \mathrm{mmol}$.

\section{MP-AES Quantification of the Number of ${ }^{\text {nat }}$ r per DFO-cRGDY-PEG-C' Dot}

To quantify the number of nat $\mathrm{Zr}$ per DFO-cRGDY-PEG-C' dot, $0.75 \mathrm{nmol}$ of DFO-cRGDYPEG-C ${ }^{\prime}$ dots were mixed with excess ${ }^{\text {nat }} \mathrm{ZrCl}_{4}(15 \mathrm{nmol})$ at $37{ }^{\circ} \mathrm{C}$ for $60 \mathrm{~min}$. The final labeling $\mathrm{pH}$ was kept at 7-7.5. After labeling, the mixture was combined with EDTA and incubated for more than $30 \mathrm{~min}$ to eliminate any nonspecific ${ }^{\mathrm{nat}} \mathrm{ZrCl}_{4}$. The sample was then purified with a PD-10 column. The amount of total labeled ${ }^{\mathrm{nat}} \mathrm{Zr}$ was then measured using MP-AES. Samples were prepared by a 10x dilution of natZr-labeled DFO-cRGDY-PEG-C' dot in $\mathrm{H}_{2} \mathrm{O}$ into $0.1 \mathrm{M} \mathrm{HCl}$ for a final matrix concentration of $0.09 \mathrm{M} \mathrm{HCl}$. The instrument was calibrated with increasing concentrations of $\mathrm{ZrCl}_{4}$ in $0.09 \mathrm{M} \mathrm{HCl}$ with a $3 \mathrm{~s}$ signal integration time and automatic background compensation. The 343.823 and $339.198 \mathrm{~nm} \mathrm{Zr}$ atomic emission wavelengths were used for calibration and analysis. The number of ${ }^{\text {nat }} \mathrm{Zr}$ per DFO-cRGDY-PEG-C ${ }^{\prime}$ dots was calculated using the following equation:

$$
\text { no. } \text { of }^{\text {nat }} \mathrm{Zr} \text { per particle }=\frac{\text { no. of }{ }^{\text {nat }} \mathrm{Zr}}{\text { no. of cRGDY }-\mathrm{PEG}-\mathrm{C}^{\prime} \mathrm{dot}}
$$

Because excess ${ }^{\text {nat }} \mathrm{ZrCl}_{4}$ was used for labeling, the number of ${ }^{\text {nat }} \mathrm{Zr}$ per ${ }^{\text {nat }} \mathrm{Zr-DFO-cRGDY-}$ PEG-C ${ }^{\prime}$ dot should roughly be equal to the number of accessible DFO per DFO-cRGDYPEG-C $C^{\prime}$ dot. Similar procedures were used for the quantification of the number of nat $\mathrm{Zr}$-percRGDY-PEG-C' ${ }^{\prime}$ dots (chelator-free radiolabeling method).

\section{Blood Circulation Half-Life Evaluations}

To estimate the blood circulation half-lives of both ${ }^{89} \mathrm{Zr}$-labeled cRGDY-PEG-C ${ }^{\prime}$ dot probes, healthy female athymic nu/nu mice (6-8 weeks old, Taconic Farms Inc.) were i.v.injected with radioactive particles. Blood sampling was performed at various postinjection time points, and these radioactive samples were counted using an Automatic Wizard ${ }^{2} \gamma$ Counter (PerkinElmer). Blood uptake values were presented as a percentage of the injected dose per gram (\% ID/g) and fit with a two-compartment model by using Prism 7 software.

\section{In Vitro and in Vivo Radio-Stability Studies}

To study in vitro radio stability, both chelator-free and chelator-based ${ }^{89} \mathrm{Zr}$-labeled cRGDYPEG-C $C^{\prime}$ dot probes $(\sim 100 \mu \mathrm{Ci}$ in $100 \mu \mathrm{L}$ PBS) were kept in PBS $(1 \times, 900 \mu \mathrm{L})$ and human serum $(1 \times, 900 \mu \mathrm{L})$ at $37^{\circ} \mathrm{C}$ under stirring at $650 \mathrm{rpm}$. Radiochemical purity was measured over a 5-day period by ITLC at various time points from the end of synthesis. The measurement was repeated three times at each time point. For in vivo radio stability, healthy female athymic nu/nu mice (6-8 weeks old, Taconic Farms Inc.) were injected with $\sim 200$ $\mu \mathrm{Ci}(\sim 7.4 \mathrm{MBq})$ of chelator-free (or chelator-based) ${ }^{89} \mathrm{Zr}$-labeled cRGDY-PEG-C ${ }^{\prime}$ dots. Whole blood was collected at 2, 24, and $48 \mathrm{~h}$ postinjection, the plasma fraction (which contained $>98 \%$ of the ${ }^{89} \mathrm{Zr}$-labeled cRGDY-PEG-C ${ }^{\prime}$ dots) was separated from the whole blood at different postinjection time points by centrifugation at $8000 \mathrm{rpm}$ for $10 \mathrm{~min}$ and used to test the radiopurity. The nonspecific association of ${ }^{89} \mathrm{Zr}$-labeled cRGDY-PEG-C' 
dots to the red blood cells was estimated to be less than $2 \%$. The percentage of the intact ${ }^{89} \mathrm{Zr}$-labeled cRGDY-PEG-C' ${ }^{\prime}$ dots was then measured by using ITLC with the plates analyzed on a Bioscan AR-2000 radio-TLC plate reader using Winscan Radio-TLC software (Bioscan Inc., Washington, DC). The measurement was repeated three times at each time point.

\section{Animal Models and Tumor Inoculation}

All animal experiments were done in accordance with protocols approved by the Institutional Animal Care and Use Committee of Memorial Sloan-Kettering Cancer Center and followed NIH guidelines for animal welfare. M21 and M21-L xenografts were generated by coinjecting equal volumes of cells $\left(\sim 5 \times 10^{6}\right.$ cells/100 $\left.\mu \mathrm{L}\right)$ and Matrigel subcutaneously into the hind legs of female athymic nu/nu mice (6-8 weeks old, Taconic Farms Inc.). Average tumor volumes of $200 \mathrm{~mm}^{3}$ were used for all studies.

\section{Dosimetry}

Time-activity curves derived for each tissue were analytically integrated, accounting for radioactive decay, to yield the corresponding cumulative activity. Organ absorbed doses were then calculated by multiplying the cumulative activity by the ${ }^{89} \mathrm{Zr}$ equilibrium dose constant for nonpenetrating radiations (positrons), assuming complete local absorption of such radiations and ignoring the contribution of penetrating radiations (i.e., $\gamma$-rays). Mouse normal organ cumulated activities were converted to human normal organ cumulated activities by taking into account differences in total-body and organ masses between mice and humans (assuming 70-kg standard human). Calculated human normal-organ cumulated activities were entered into the OLINDA dosimetry program to compute standard human organ absorbed doses using formalism of the Medical Internal Dosimetry Committee of the Society of Nuclear Medicine. ${ }^{46}$ This human dosimetry model is a normal (i.e., tumor-free) anatomic model.

\section{In Vivo Static PET, Dynamic PET Imaging, and ex Vivo Biodistribution Studies}

For static PET imaging, tumor-bearing mice $(n=3)$ were i.v.-injected with $200-300 \mu \mathrm{Ci}$ (7.4-11.1 MBq) cRGDY-PEG- $\left.{ }^{89} \mathrm{Zr}\right] \mathrm{C}^{\prime}$ dots (labeling condition: $4 \mathrm{nmol} / \mathrm{mCi}, \mathrm{pH} 8,75{ }^{\circ} \mathrm{C}$, $120 \mathrm{~min}$ ) or ${ }^{89} \mathrm{Zr}$-DFO-cRGDY-PEG-C' dots (labeling condition: $0.75 \mathrm{nmol} / \mathrm{mCi}, \mathrm{pH} 7.4$, $37^{\circ} \mathrm{C}, 60 \mathrm{~min}$ ). Approximately $5 \mathrm{~min}$ prior to the acquisition of PET images, mice were anesthetized by inhalation of $2 \%$ isoflurane (Baxter Healthcare, Deerfield, IL)/ oxygen gas mixture and placed on the scanner bed; anesthesia was maintained using $1 \%$ isoflurane/gas mixture. PET imaging was performed in a small-animal PET scanner (Focus 120 microPET; Concorde Microsystems) at 2, 24, 48, and $72 \mathrm{~h}$ postinjection. An energy window of 350-700 $\mathrm{keV}$ and a coincidence timing window of $6 \mathrm{~ns}$ were used. Data were sorted into 2D histograms by Fourier rebinning, and transverse images were reconstructed by filtered backprojection into a $128 \times 128 \times 63\left(0.72 \times 0.72 \times 1.3 \mathrm{~mm}^{3}\right)$ matrix. The PET imaging data were normalized to correct for nonuniformity of response, dead-time count losses, positron branching ratio, and physical decay to the time of injection; no attenuation, scatter, or partial-volume averaging corrections were applied. The counting rates in the reconstructed images were converted to activity concentrations (percentage injected dose per gram of tissue, $\% \mathrm{ID} / \mathrm{g}$ ) by use of a system calibration factor derived from the imaging of a mouse- 
sized water-equivalent phantom containing ${ }^{89} \mathrm{Zr}$. Region-of-interest (ROI) analyses of the PET data were performed using IRW software.

For dynamic PET scanning, healthy mice were i.v. injected with $\sim 400 \mu \mathrm{Ci}(\sim 14.8 \mathrm{MBq})$ of cRGDY-PEG- $\left.{ }^{89} \mathrm{Zr}\right] \mathrm{C}^{\prime}$ dots or ${ }^{89} \mathrm{Zr}$-DFO-cRGDY-PEG-C ${ }^{\prime}$ dots. A 60 min dynamic scan was performed in a small-animal PET scanner (Focus 120 microPET; Concorde Microsystems) and framed into 46 frames: $12 \times 5,6 \times 10,6 \times 30,10 \times 60,6 \times 150$, and $5 \times 300 \mathrm{~s}$. Image reconstruction and ROI analysis were performed using IRW software and presented as $\% \mathrm{ID} / \mathrm{g}$.

For biodistribution studies, tumor-bearing $(n=3)$ mice were injected with $\sim 100 \mu \mathrm{Ci}(\sim 3.7$ $\mathrm{MBq})$ cRGDY-PEG- $\left.{ }^{89} \mathrm{Zr}\right] \mathrm{C}^{\prime}$ dots or ${ }^{89} \mathrm{Zr}$-DFO-cRGDY-PEG-C ${ }^{\prime}$ dots. Accumulated activity in major intraparenchymal organs was assayed at $24 \mathrm{~h}$ using an Automatic Wizard ${ }^{2}$ $\gamma$-Counter (PerkinElmer) and presented as $\% \mathrm{ID} / \mathrm{g}($ mean $\pm \mathrm{SD})$.

\section{Statistics}

All comparisons were based on statistical significance from pairwise $t$ tests from an analysis of variance model based on three replicates.

\section{Supplementary Material}

Refer to Web version on PubMed Central for supplementary material.

\section{Acknowledgments}

Funding

This study was funded by grants from the National Institutes of Health (1R01CA161280-01A1 to M.B. and U.W.; 1U54 CA199081-01 to M.B. and U.W.) and Sloan Kettering Institute (Core Grant P30 CA008748CCSG).

\section{References}

1. Smith BR, Gambhir SS. Nanomaterials for in Vivo Imaging. Chem Rev. 2017; 117:901-986. [PubMed: 28045253]

2. Thakor AS, Gambhir SS. Nanooncology: the Future of Cancer Diagnosis and Therapy. Ca-Cancer J Clin. 2013; 63:395-418. [PubMed: 24114523]

3. Anselmo AC, Mitragotri S. A Review of Clinical Translation of Inorganic Nanoparticles. AAPS J. 2015; 17:1041-54. [PubMed: 25956384]

4. Anselmo AC, Mitragotri S. Nanoparticles in the Clinic. Bioeng Transl Med. 2016; 1:10-29.

5. Wilhelm S, Tavares AJ, Dai Q, Ohta S, Audet J, Dvorak HF, Chan WCW. Analysis of Nanoparticle Delivery to Tumours. Nat Rev Mater. 2016; 1:16014.

6. Zhang YN, Poon W, Tavares AJ, McGilvray ID, Chan WC. Nanoparticle-Liver Interactions: Cellular Uptake and Hepatobiliary Elimination. J Controlled Release. 2016; 240:332-348.

7. D’Mello SR, Cruz CN, Chen ML, Kapoor M, Lee SL, Tyner KM. The Evolving Landscape of Drug Products Containing Nanomaterials in the United States. Nat Nanotechnol. 2017; 12:523-529. [PubMed: 28436961]

8. Soo Choi H, Liu W, Misra P, Tanaka E, Zimmer JP, Itty Ipe B, Bawendi MG, Frangioni JV. Renal Clearance of Quantum Dots. Nat Biotechnol. 2007; 25:1165-1170. [PubMed: 17891134]

9. Choi HS, Liu W, Liu F, Nasr K, Misra P, Bawendi MG, Frangioni JV. Design Considerations for Tumour-Targeted Nano-particles. Nat Nanotechnol. 2010; 5:42-7. [PubMed: 19893516] 
10. Zhou C, Long M, Qin Y, Sun X, Zheng J. Luminescent Gold Nanoparticles with Efficient Renal Clearance. Angew Chem, Int Ed. 2011; 50:3168-72.

11. Zhou C, Hao G, Thomas P, Liu J, Yu M, Sun S, Oz OK, Sun X, Zheng J. Near-infrared Emitting Radioactive Gold Nanoparticles with Molecular Pharmacokinetics. Angew Chem, Int Ed. 2012; 51:10118-22.

12. Liu J, Yu M, Ning X, Zhou C, Yang S, Zheng J. PEGylation and Zwitterionization: Pros and Cons in the Renal Clearance and Tumor Targeting of Near-IR-Emitting Gold Nano-particles. Angew Chem, Int Ed. 2013; 52:12572-6.

13. Zhou M, Li J, Liang S, Sood AK, Liang D, Li C. CuS Nanodots with Ultrahigh Efficient Renal Clearance for Positron Emission Tomography Imaging and Image-Guided Photothermal Therapy. ACS Nano. 2015; 9:7085-96. [PubMed: 26098195]

14. Chen F, Goel S, Hernandez R, Graves SA, Shi S, Nickles RJ, Cai W. Dynamic Positron Emission Tomography Imaging of Renal Clearable Gold Nanoparticles. Small. 2016; 12:2775-82. [PubMed: 27062146]

15. Burns AA, Vider J, Ow H, Herz E, Penate-Medina O, Baumgart M, Larson SM, Wiesner U, Bradbury M. Fluorescent Silica Nanoparticles with Efficient Urinary Excretion for Nanomedicine. Nano Lett. 2009; 9:442-448. [PubMed: 19099455]

16. Benezra M, Penate-Medina O, Zanzonico PB, Schaer D, Ow H, Burns A, DeStanchina E, Longo V, Herz E, Iyer S, Wolchok J, Larson SM, Wiesner U, Bradbury MS. Multimodal Silica Nanoparticles are Effective Cancer-Targeted Probes in a Model of Human Melanoma. J Clin Invest. 2011; 121:2768-80. [PubMed: 21670497]

17. Phillips E, Penate-Medina O, Zanzonico PB, Carvajal RD, Mohan P, Ye Y, Humm J, Gonen M, Kalaigian H, Schoder H, Strauss HW, Larson SM, Wiesner U, Bradbury MS. Clinical Translation of an Ultrasmall Inorganic Optical-PET Imaging Nano-particle Probe. Sci Transl Med. 2014; 6:260ra149.

18. Ma K, Zhang DH, Cong Y, Wiesner U. Elucidating the Mechanism of Silica Nanoparticle PEGylation Processes Using Fluorescence Correlation Spectroscopies. Chem Mater. 2016; 28:1537-1545.

19. Herz E, Ow H, Bonner D, Burns A, Wiesner U. Dye Structure-Optical Property Correlations in Near-Infrared Fluorescent Core-Shell Silica Nanoparticles. J Mater Chem. 2009; 19:6341-6347.

20. Ow H, Larson DR, Srivastava M, Baird BA, Webb WW, Wiesner U. Bright and Stable Core-Shell Fluorescent Silica Nanoparticles. Nano Lett. 2005; 5:113-7. [PubMed: 15792423]

21. Ma K, Mendoza C, Hanson M, Werner-Zwanziger U, Zwanziger J, Wiesner U. Control of Ultrasmall Sub-10 nm Ligand-Functionalized Fluorescent Core-Shell Silica Nanoparticle Growth in Water. Chem Mater. 2015; 27:4119-4133.

22. Kim SE, Zhang L, Ma K, Riegman M, Chen F, Ingold I, Conrad M, Turker MZ, Gao M, Jiang X, Monette S, Pauliah M, Gonen M, Zanzonico P, Quinn T, Wiesner U, Bradbury MS, Overholtzer M. Ultrasmall Nanoparticles Induce Ferroptosis in Nutrient-Deprived Cancer Cells and Suppress Tumour Growth. Nat Nanotechnol. 2016; 11:977-985. [PubMed: 27668796]

23. Bradbury MS, Phillips E, Montero PH, Cheal SM, Stambuk H, Durack JC, Sofocleous CT, Meester RJ, Wiesner U, Patel S. Clinically-translated Silica Nanoparticles as Dual-Modality CancerTargeted Probes for Image-Guided Surgery and Interventions. Integr Biol (Camb). 2013; 5:74-86. [PubMed: 23138852]

24. Bradbury MS, Pauliah M, Zanzonico P, Wiesner U, Patel S. Intraoperative Mapping of Sentinel Lymph Node Metastases using a Clinically Translated Ultrasmall Silica Nanoparticle. Wiley Interdiscip Rev Nanomed Nanobiotechnol. 2016; 8:535-53. [PubMed: 26663853]

25. Holland JP, Divilov V, Bander NH, Smith-Jones PM, Larson SM, Lewis JS. ${ }^{89}$ Zr-DFO-J591 for Immunopet of Prostate-Specific Membrane Antigen Expression In Vivo. J Nucl Med. 2010; 51:1293-300. [PubMed: 20660376]

26. Holland JP, Evans MJ, Rice SL, Wongvipat J, Sawyers CL, Lewis JS. Annotating MYC Status with ${ }^{89}$ Zr-transferrin Imaging. Nat Med. 2012; 18:1586-91. [PubMed: 23001181]

27. Perez-Medina C, Tang J, Abdel-Atti D, Hogstad B, Merad M, Fisher EA, Fayad ZA, Lewis JS, Mulder WJ, Reiner T. PET Imaging of Tumor-Associated Macrophages with 89Zr-Labeled HighDensity Lipoprotein Nanoparticles. J Nucl Med. 2015; 56:1272-7. [PubMed: 26112022] 
28. Clinicaltrials.gov. https://clinicaltrials.gov/ct2/results?term $=89 \mathrm{Zr} \& \mathrm{cntry} 1=\mathrm{NA} \% 3$ AUS (accessed September 6, 2017)

29. Lee FT, Scott AM. Immuno-PET for Tumor Targeting. J Nucl Med. 2003; 44:1282. [PubMed: 12902419]

30. Chen F, Goel S, Valdovinos HF, Luo H, Hernandez R, Barnhart TE, Cai W. In Vivo Integrity and Biological Fate of Chelator-Free Zirconium-89-Labeled Mesoporous Silica Nanopar-ticles. ACS Nano. 2015; 9:7950-9. [PubMed: 26213260]

31. Shaffer TM, Wall MA, Harmsen S, Longo VA, Drain CM, Kircher MF, Grimm J. Silica Nanoparticles as Substrates for Chelator-Free Labeling of Oxophilic Radioisotopes. Nano Lett. 2015; 15:864-8. [PubMed: 25559467]

32. Goel S, Chen F, Ehlerding EB, Cai W. Intrinsically Radiolabeled Nanoparticles: an Emerging Paradigm. Small. 2014; 10:3825-30. [PubMed: 24978934]

33. Chen F, Ellison PA, Lewis CM, Hong H, Zhang Y, Shi S, Hernandez R, Meyerand ME, Barnhart TE, Cai W. Chelator-free Synthesis of a Dual-Modality PET/MRI Agent. Angew Chem, Int Ed. 2013; 52:13319-23.

34. Ellison PA, Chen F, Goel S, Barnhart TE, Nickles RJ, DeJesus OT, Cai W. Intrinsic and Stable Conjugation of Thiolated Mesoporous Silica Nanoparticles with Radioarsenic. ACS Appl Mater Interfaces. 2017; 9:6772-6781. [PubMed: 28165700]

35. Chakravarty R, Valdovinos HF, Chen F, Lewis CM, Ellison PA, Luo H, Meyerand ME, Nickles RJ, Cai W. Intrinsically Germanium-69-Labeled Iron Oxide Nanoparticles: Synthesis and In-Vivo Dual-Modality PET/MR Imaging. Adv Mater. 2014; 26:5119-23. [PubMed: 24944166]

36. Chen F, Valdovinos HF, Hernandez R, Goel S, Barnhart TE, Cai W. Intrinsic Radiolabeling of Titanium-45 using Mesoporous Silica Nanoparticles. Acta Pharmacol Sin. 2017; 38:907-913. [PubMed: 28414201]

37. Zhuravlev LT. The surface chemistry of amorphous silica. Zhuravlev model. Colloids Surf, A. 2000; 173:1-38.

38. Larson DR, Ow H, Vishwasrao HD, Heikal AA, Wiesner U, Webb WW. Silica Nanoparticle Architecture Determines Radiative Properties of Encapsulated Fluorophores. Chem Mater. 2008; 20:2677-2684.

39. Lin YS, Abadeer N, Hurley KR, Haynes CL. Ultrastable, Redispersible, Small, and Highly Organomodified Mesoporous Silica Nanotherapeutics. J Am Chem Soc. 2011; 133:20444-57. [PubMed: 22050408]

40. Holland JP, Sheh Y, Lewis JS. Standardized Methods for the Production of High Specific-Activity Zirconium-89. Nucl Med Biol. 2009; 36:729-39. [PubMed: 19720285]

41. Goel S, Chen F, Luan S, Valdovinos HF, Shi S, Graves SA, Ai F, Barnhart TE, Theuer CP, Cai W. Engineering Intrinsically Zirconium-89 Radiolabeled Self-Destructing Mesoporous Silica Nanostructures for In Vivo Biodistribution and Tumor Targeting Studies. Adv Sci (Weinh). 2016; 3:1600122. [PubMed: 27980987]

42. Meijs WE, Herscheid JD, Haisma HJ, Pinedo HM. Evaluation of Desferal as a Bifunctional Chelating Agent for Labeling Antibodies with Zr-89. Int J Rad Appl Instrum A. 1992; 43:1443-7. [PubMed: 1334954]

43. Ma K, Wiesner U. Modular and Orthogonal Post-PEGylation Surface Modifications by Insertion Enabling Penta-functional Ultra-small Organic-Silica Hybrid Nanoparticles. Chem Mater. 2017; 29:6840-55.

44. Gambhir SS. Molecular Imaging of Cancer with Positron Emission Tomography. Nat Rev Cancer. 2002; 2:683-93. [PubMed: 12209157]

45. Abou DS, Ku T, Smith-Jones PM. In Vivo Biodistribution and Accumulation of ${ }^{89} \mathrm{Zr}$ in Mice. Nucl Med Biol. 2011; 38:675-81. [PubMed: 21718943]

46. Stabin MG, Sparks RB, Crowe E. OLINDA/EXM: the Second-Generation Personal Computer Software for Internal Dose Assessment in Nuclear Medicine. J Nucl Med. 2005; 46:1023. [PubMed: 15937315]

47. Laforest R, Lapi SE, Oyama R, Bose R, Tabchy A, Marquez-Nostra BV, Burkemper J, Wright BD, Frye J, Frye S, Siegel BA, Dehdashti F. [ ${ }^{89} \mathrm{Zr}$ ]Trastuzumab: Evaluation of Radiation Dosimetry, 
Safety, and Optimal Imaging Parameters in Women with HER2-Positive Breast Cancer. Mol Imaging Biol. 2016; 18:952-959. [PubMed: 27146421]

48. Bjornmalm M, Faria M, Caruso F. Increasing the Impact of Materials in and beyond Bio-Nano Science. J Am Chem Soc. 2016; 138:13449-13456. [PubMed: 27672703]

49. McNeil SE. Evaluation of Nanomedicines: Stick to the Basics. Nat Rev Mater. 2016; 1:16073.

50. Torrice M. Does Nanomedicine Have a Delivery Problem? ACS Cent Sci. 2016; 2:434-7. [PubMed: 27504489]

51. Wilhelm S, Tavares AJ, Chan WCW. Reply to "Evaluation of Nanomedicines: Stick to the Basics". Nat Rev Mater. 2016; 1:16074.

52. Chan WCW. Nanomedicine 2.0. Acc Chem Res. 2017; 50:627-632. [PubMed: 28945418]

53. van der Meel R, Lammers T, Hennink WE. Cancer Nanomedicines: Oversold or Underappreciated? Expert Opin Drug Delivery. 2017; 14:1-5. 
a)

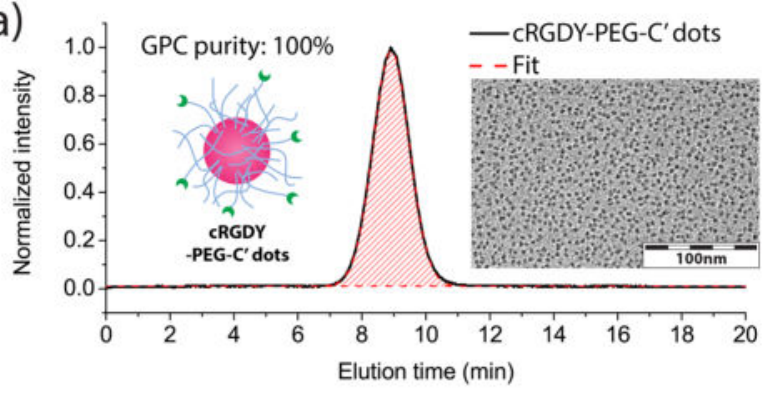

b)
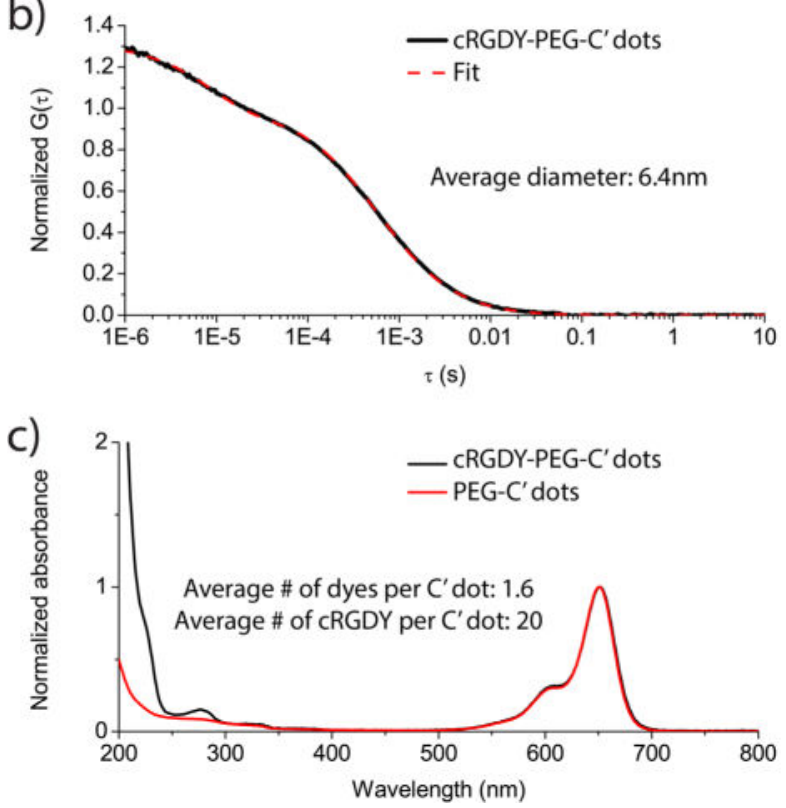

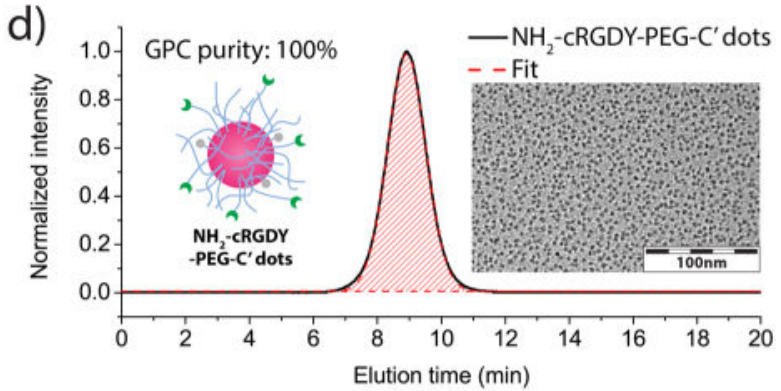

e)
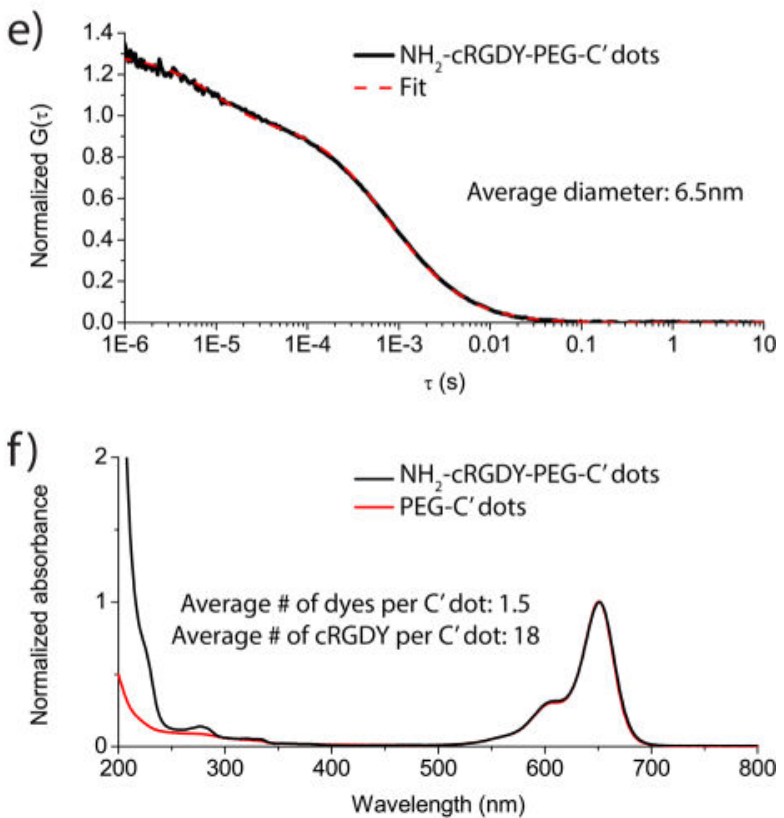

Figure 1.

Characterization of cRGDY-PEG-C $\mathrm{C}^{\prime}$ dots and $\mathrm{NH}_{2}$-cRGDY-PEG-C $\mathrm{C}^{\prime}$ dots. GPC elugram with fit (a), FCS Characterization of cRGDY-PEG-C' dots and $\mathrm{NH}_{2}$-cRGDY-PEG-C ${ }^{\prime}$ dots. GPC elugram with fit (a), FCS correlation curve with fit (b), and UV-vis absorbance spectra (c) of cRGDY-PEG-C' ${ }^{\prime}$ dots as compared to those of PEG-C ${ }^{\prime}$ dots. GPC elugram with fit (d), FCS correlation curve with fit (e), and UV-vis absorbance spectra (f) of aminefunctionalized $\mathrm{NH}_{2}$-cRGDY-PEG-C ${ }^{\prime}$ dots as compared to those of PEG-C ${ }^{\prime}$ dots. 
a)

$\mathrm{pH} 8,75^{\circ} \mathrm{C}$ (Chelator-free)

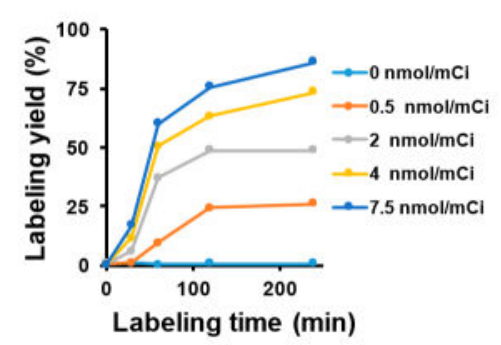

d)

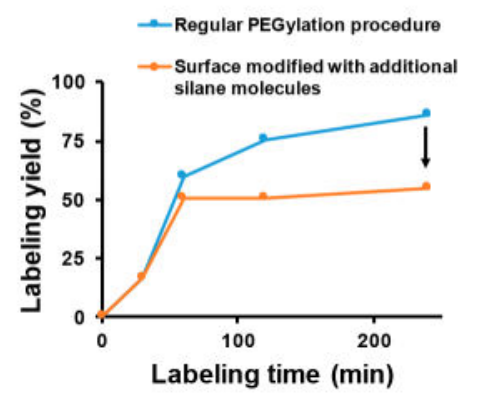

b)

$7.5 \mathrm{nmol} / \mathrm{mCi}, 75^{\circ} \mathrm{C}$ (Chelator-free)

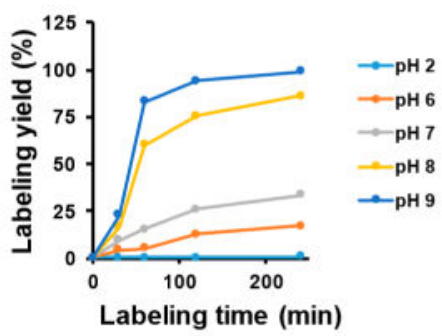

e)

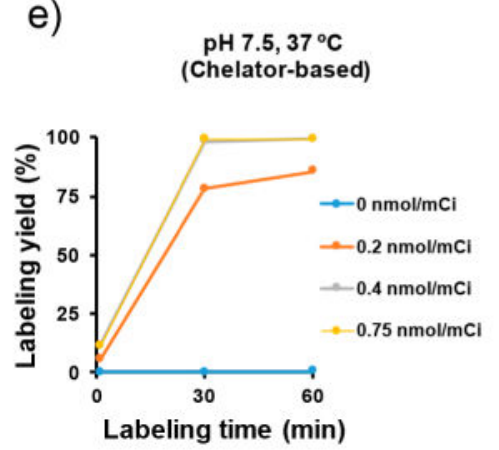

C) $\quad \begin{aligned} & 7.5 \mathrm{nmol} / \mathrm{mCi}, \mathrm{pH} \\ & \text { (Chelator-free) }\end{aligned}$

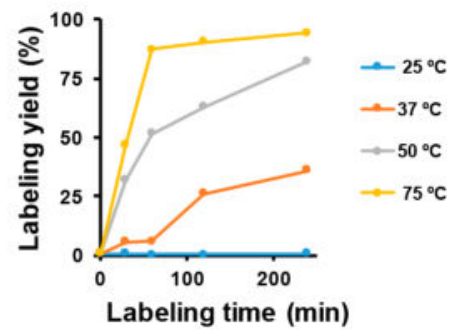

f)

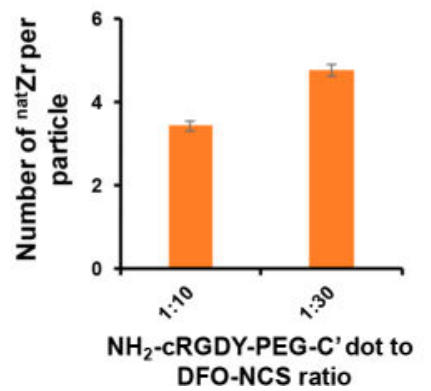

Figure 2.

Chelator-free and chelator-based ${ }^{89} \mathrm{Zr}$ radiolabeling studies. (a) Concentration-dependent chelator-free ${ }^{89} \mathrm{Zr}$ labeling of cRGDY-PEG-C ${ }^{\prime}$ dots. Labeling temperature was set to $75^{\circ} \mathrm{C}$; labeling $\mathrm{pH}$ was set to 8 , and $\mathrm{C}^{\prime} \operatorname{dot}(\mathrm{nmol})$ to ${ }^{89} \mathrm{Zr}(\mathrm{mCi})$ ratio was in the range of 0-7.5 $\mathrm{nmol} / \mathrm{mCi}$. (b) $\mathrm{pH}-$ Dependent chelator-free ${ }^{89} \mathrm{Zr}$ labeling. Labeling temperature: $75{ }^{\circ} \mathrm{C} ; \mathrm{C}^{\prime}$ dot to ${ }^{89} \mathrm{Zr}$ ratio: $7.5 \mathrm{nmol} / \mathrm{mCi}$; labeling $\mathrm{pH}$ range: $2-9$. (c) Temperature-dependent chelator-free ${ }^{89} \mathrm{Zr}$ labeling. Labeling pH: 8; $\mathrm{C}^{\prime}$ dot to ${ }^{89} \mathrm{Zr}$ ratio: $7.5 \mathrm{nmol} / \mathrm{mCi}$; labeling temperature range: $25-75^{\circ} \mathrm{C}$. (d) Chelator-free ${ }^{89} \mathrm{Zr}$ labeling comparison between $\mathrm{C}^{\prime}$ dots with regular PEGylation procedures and PEGylated $\mathrm{C}^{\prime}$ dots further modified with additional small silane molecules (i.e., DEDMS: diethoxy dimethyl silane). Labeling temperature: $75^{\circ} \mathrm{C}$; labeling pH: $8 ; \mathrm{C}^{\prime}$ dot to ${ }^{89} \mathrm{Zr}$ ratio: $7.5 \mathrm{nmol} / \mathrm{mCi}$. (e) Concentration-dependent chelator-based ${ }^{89} \mathrm{Zr}$ labeling of DFO-cRGDY-PEG-C ${ }^{\prime}$ dots. Labeling temperature: $37{ }^{\circ} \mathrm{C}$; labeling pH: 7.5; $\mathrm{C}^{\prime}$ dot to ${ }^{89} \mathrm{Zr}$ ratio range: $0-0.75 \mathrm{nmol} / \mathrm{mCi}$. (f) MP-AES testing of the number of natZr per DFO-cRGDY-PEG-C ${ }^{\prime}$ dot particles synthesized with varied particle to DFO-NCS ratios. The radiolabeling yield was evaluated once per time point (a-e). MP-AES measurements of nat $\mathrm{Zr}$ concentrations were repeated in triplicate (f). 
a)
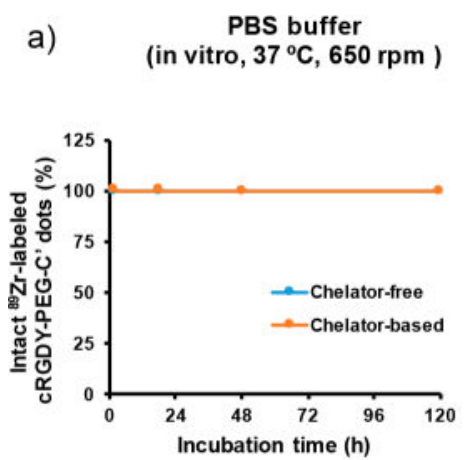

d)

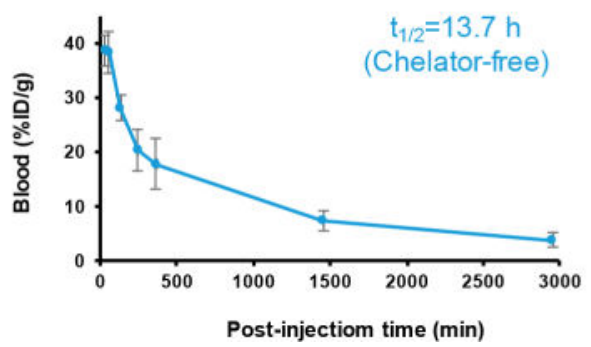

c)

Mouse whole blood (in vivo, i.v.)
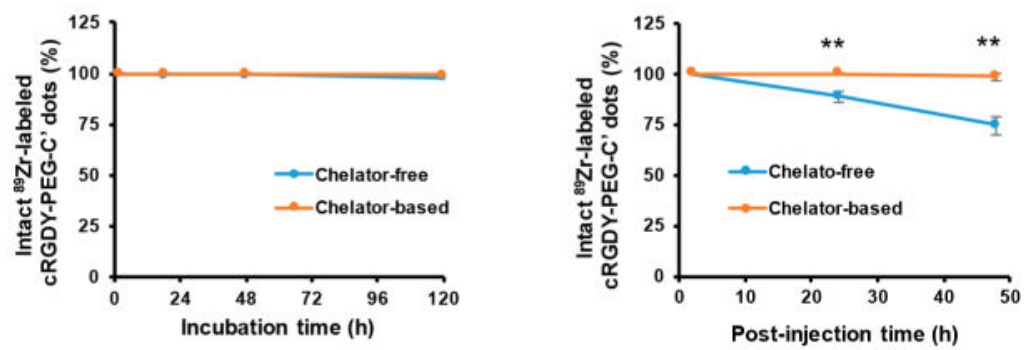

e)

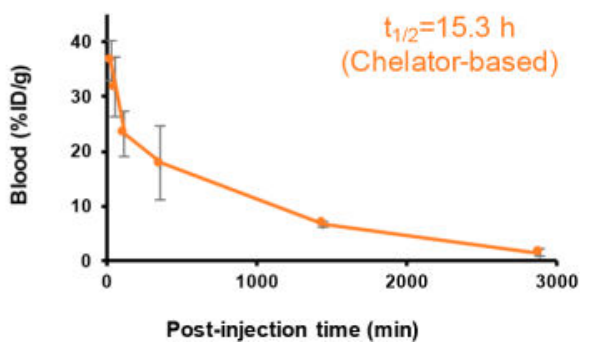

Figure 3.

Comparison of chelator-free and chelator-based ${ }^{89} \mathrm{Zr}$-labeled $\mathrm{C}^{\prime}$ dot properties.

Radiostability of ${ }^{89} \mathrm{Zr}$-labeled cRGDY-PEG-C ${ }^{\prime}$ dots in (a) PBS, $37{ }^{\circ} \mathrm{C}$ under stirring at 650 $\mathrm{rpm}$, (b) human serum, $37{ }^{\circ} \mathrm{C}$ under stirring at $650 \mathrm{rpm}$, and (c) in vivo, i.v.-injected into healthy female athymic nu/nu mice (6-8 weeks old). Plasma (which contained $>98 \%$ of the ${ }^{89} \mathrm{Zr}$-labeled cRGDY-PEG-C ${ }^{\prime}$ dots) was separated from whole blood at different postinjection time points and used to assess radiopurity. The nonspecific association of ${ }^{89} \mathrm{Zr}$ labeled cRGDY-PEG-C ${ }^{\prime}$ dots to red blood cells was estimated to be less than $2 \%$. Blood circulation half-life fitting for (d) chelator-free ${ }^{89} \mathrm{Zr}$-labeled cRGDY-PEG-C ${ }^{\prime}$ dots $(n=3)$ and (e) chelator-based ${ }^{89} \mathrm{Zr}$-labeled cRGDY-PEG-C ${ }^{\prime}$ dots $(n=3)$. $\left({ }^{* *} p<0.005\right)$. For each time point, radiopurity of ${ }^{89} \mathrm{Zr}$-labeled cRGDY-PEG-C' dots was evaluated in triplicate. Note: error bars in panels $\mathrm{a}$ and $\mathrm{b}$ are smaller than the size of the data points. 
a)
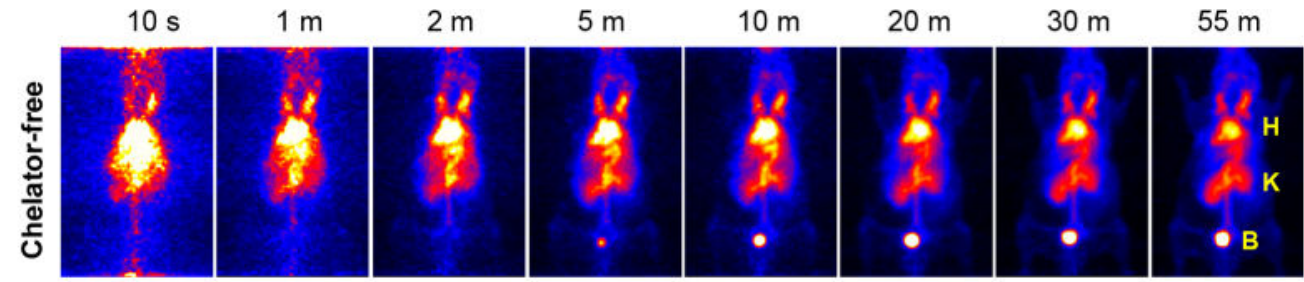

b)
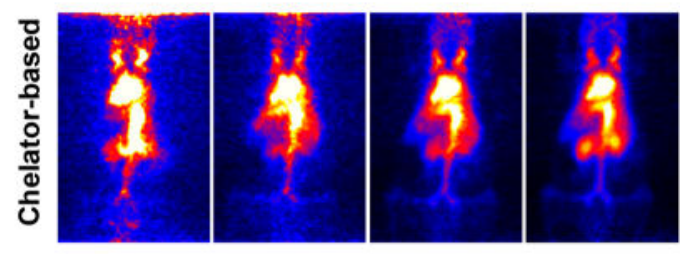

c)

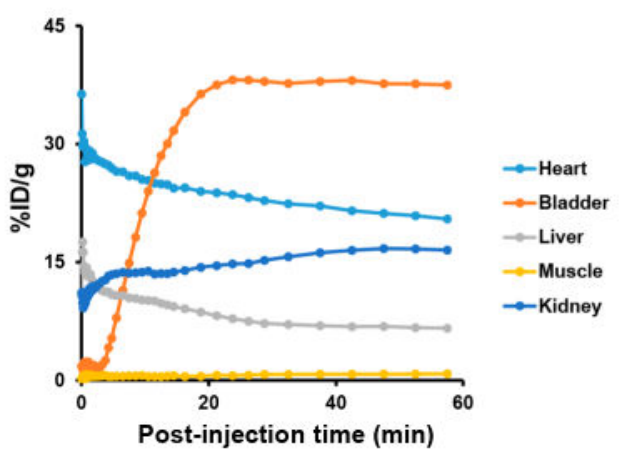

d)

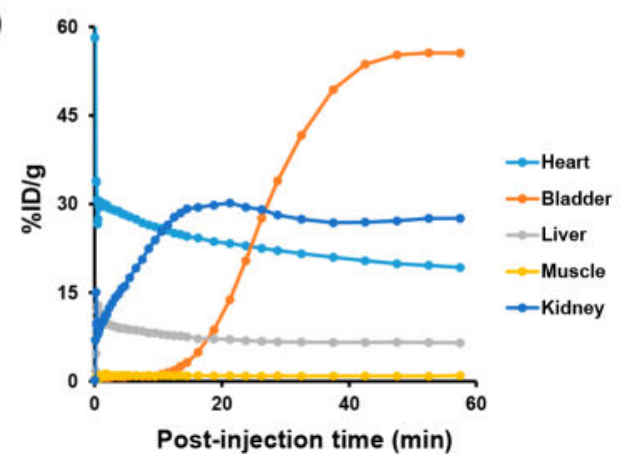

Figure 4.

Comparison of dynamic PET imaging results in mice for chelator-free and chelatorbased ${ }^{89} \mathrm{Zr}$-labeled $\mathrm{C}^{\prime}$ dots. (a) Chelator-free ${ }^{89} \mathrm{Zr}$-labeled cRGDY-PEG- $\mathrm{C}^{\prime}$ dots and (b) chelator-based ${ }^{89} \mathrm{Zr}$-labeled cRGDY-PEG-C' ${ }^{\prime}$ dots. H: heart; K: kidney; B: bladder. The first 60 min time- activity curves for major organs (i.e., heart, bladder, liver, muscle, and kidney) in mice i.v.-injected with (c) chelator-free ${ }^{89} \mathrm{Zr}$-labeled cRGDY-PEG- $\left.{ }^{89} \mathrm{Zr}\right] \mathrm{C}^{\prime}$ dots and (d) chelator-based ${ }^{89} \mathrm{Zr}$-labeled ${ }^{89} \mathrm{Zr}$-DFO-cRGDY-PEG-C ${ }^{\prime}$ dots. All images in panels a and b are coronal MIP PET images. For each group, a representative mouse was used to acquire dynamic PET data. 
a)

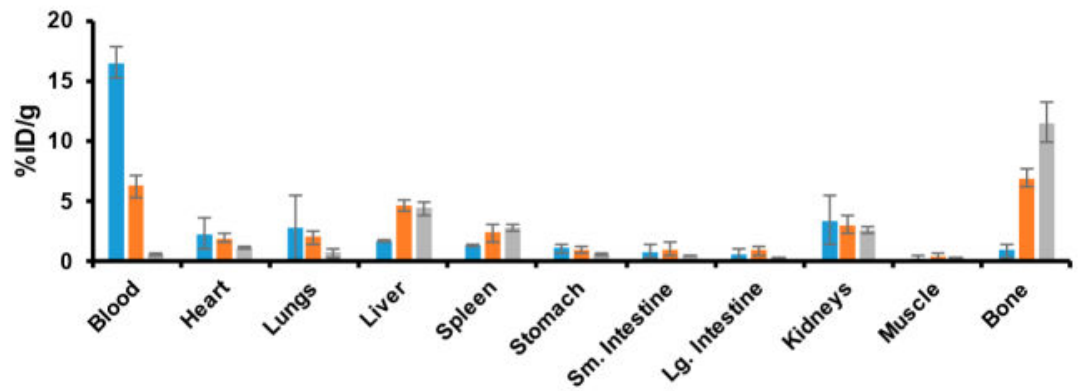

b)

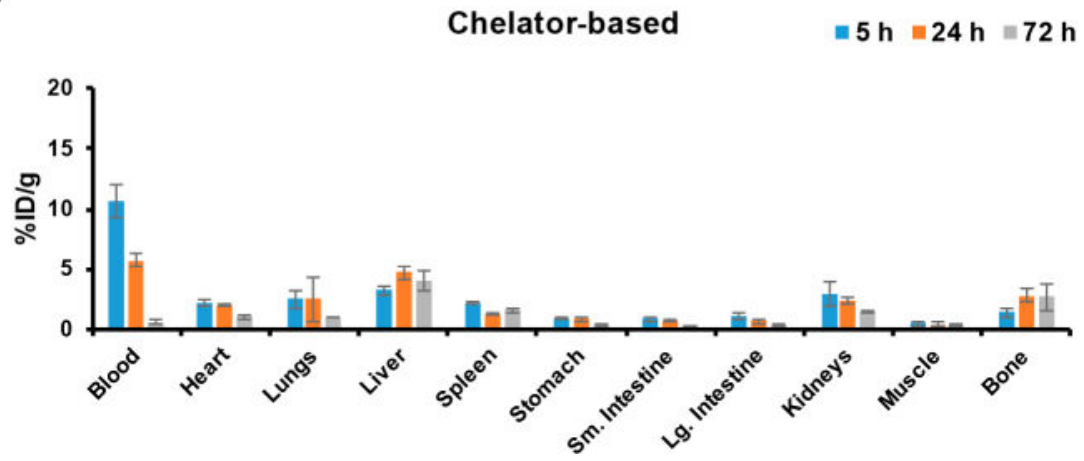

c)

= Chelator-based = Chelator-free

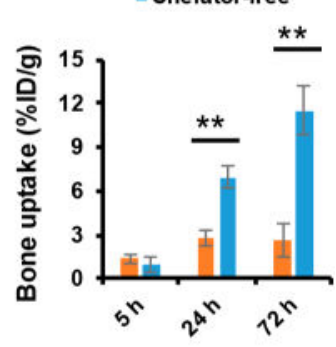

Post-injection time

Figure 5.

Biodistribution studies in mice for chelator-free and chelator-based ${ }^{89} \mathrm{Zr}$-labeled $\mathrm{C}^{\prime}$ dots. (a) Chelator-free ${ }^{89} \mathrm{Zr}$-labeled cRGDY-PEG-[ $\left.{ }^{89} \mathrm{Zr}\right] \mathrm{C}^{\prime}$ dots and (b) chelator-based ${ }^{89} \mathrm{Zr}$ labeled ${ }^{89} \mathrm{Zr}$-DFO-cRGDY-PEG-C ${ }^{\prime}$ dots in healthy mice $(n=3)$. (c) Comparison of timedependent bone uptake in mice injected with the ${ }^{89} \mathrm{Zr}$-labeled cRGDY-PEG-C ${ }^{\prime}$ dots $\left({ }^{* *} p<\right.$ $0.005)$. 
a)
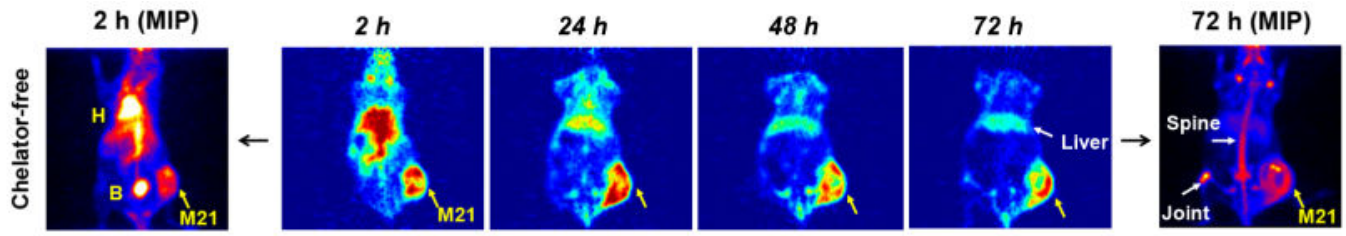

b)
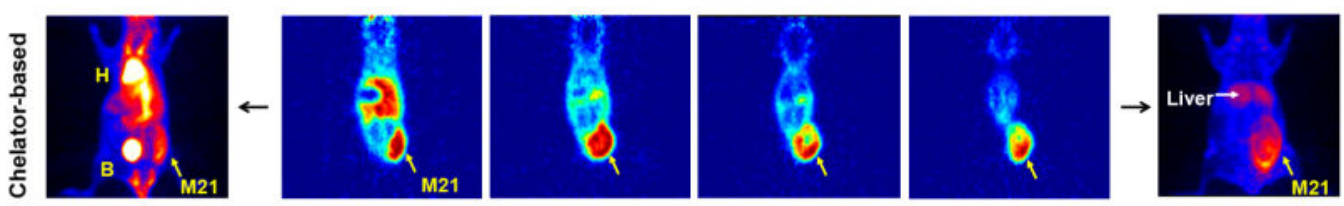

c)
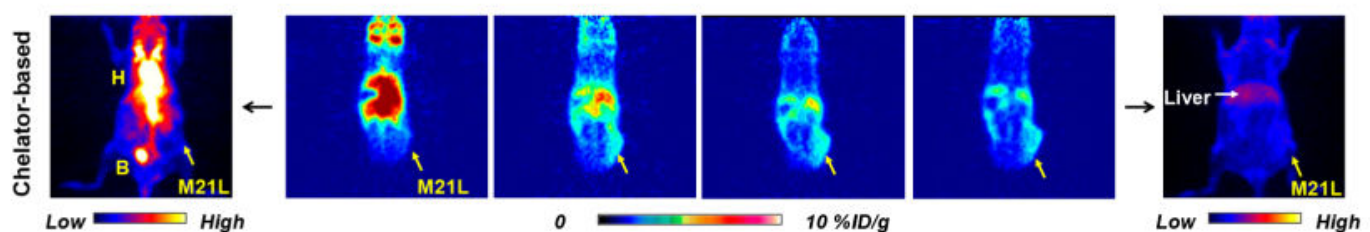

d)

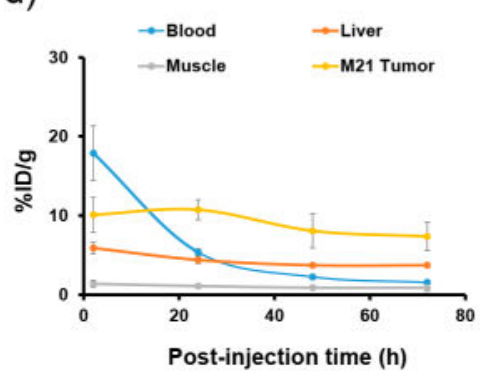

e)

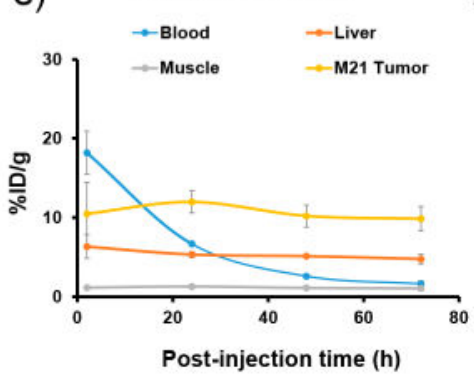

f)

Chelator-based in M21L

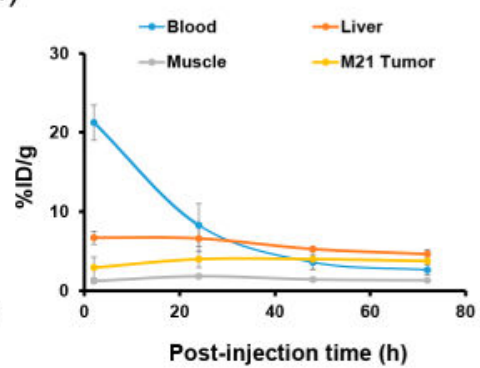

$\rightarrow$ Chelator-free in M2

g)

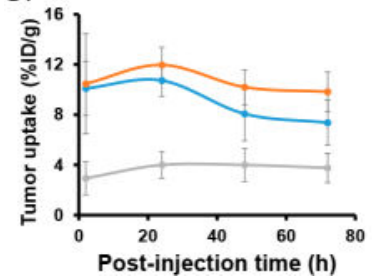

h)

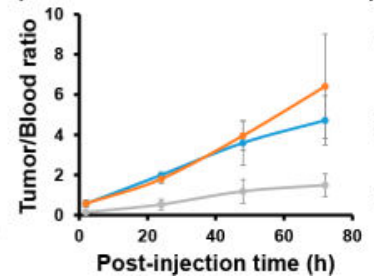

i)

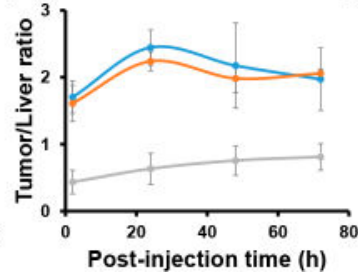

j)

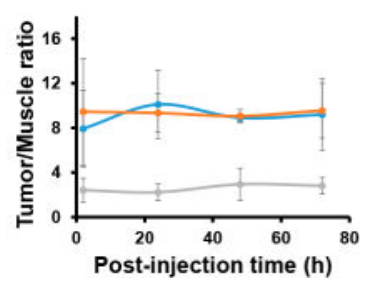

Figure 6.

In vivo tumor-targeted coronal PET images of mice and their analysis. Mice injected with (a) cRGDY-PEG- $\left[{ }^{89} \mathrm{Zr}\right] \mathrm{C}^{\prime}$ dots, chelator-free labeling, in M21 tumor-bearing mice $(n=3)$, (b) ${ }^{89} \mathrm{Zr}$-DFO-cRGDY-PEG-C' ${ }^{\prime}$ dots, chelator-based labeling, in M21 tumor-bearing mice ( $n$ = 3), and (c) ${ }^{89} \mathrm{Zr}$-DFO-cRGDY-PEG-C ${ }^{\prime}$ dots, chelator-based labeling, in M21L tumorbearing mice $(n=3)$. MIP images at 2 and $72 \mathrm{~h}$ are presented to reveal the extended blood half-lives of the particles, renal clearance of particles into the bladder at $2 \mathrm{~h}$ postinjection, and bone and joint uptake at $72 \mathrm{~h}$ postinjection. Time activity curves showing (d) chelatorfree ${ }^{89} \mathrm{Zr}$-labeled cRGDY-PEG- $\left.{ }^{89} \mathrm{Zr}\right] \mathrm{C}^{\prime}$ dot in M21 xenografts, (e) chelator-based ${ }^{89} \mathrm{Zr}$ labeled ${ }^{89} \mathrm{Zr}$-DFO-cRGDY-PEG-C' ${ }^{\prime}$ dots in M21 xenografts, and (f) chelator-based ${ }^{89} \mathrm{Zr}$ labeled ${ }^{89} \mathrm{Zr}$-DFO-cRGDY-PEG-C' ${ }^{\prime}$ dots in M21L xenografts. Comparisons of (g) tumor uptake, (h) tumor-to-blood ratios, (i) tumor-to-liver ratios, and (j) tumor-to-muscle ratios among three groups. $N=3$ for each group. 
a)
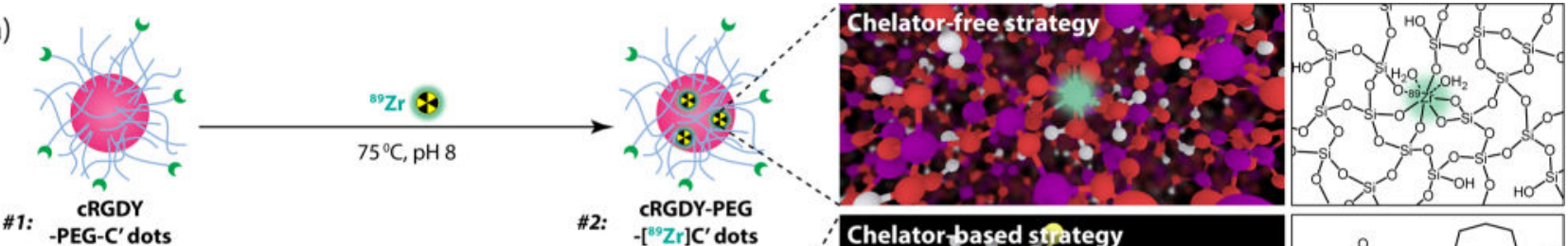

1: -PEG-C'dots

\#2: $\quad$ CRGDY-PEG

b)
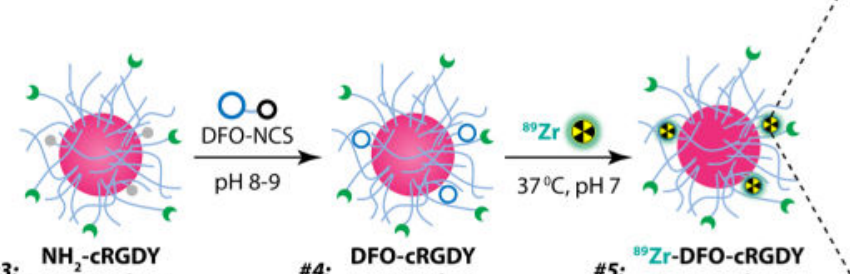

-PEG-C' dots

$\# 5: \stackrel{{ }^{89} \mathrm{Zr} \text {-DFO-CRGD }}{\quad \text {-PEG-C' dots }}$
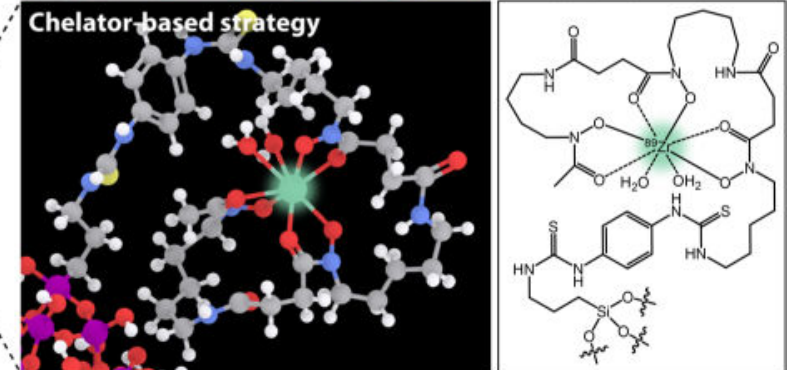

Scheme $1 .{ }^{89} \mathrm{Zr}$-Radiolabeling Strategies of cRGDY-PEG-C ${ }^{\prime}$ Dots $^{\boldsymbol{a}}$

${ }^{a}$ (a) Chelator-free strategy: the surface and/or internal deprotonated silanol groups $\left(-\mathrm{Si}-\mathrm{O}^{-}\right)$ from the (1) cRGDY-PEG- $\mathrm{C}^{\prime}$ dots are functioning as the inherent oxygen donors (or hard Lewis bases) for the successful labeling of ${ }^{89} \mathrm{Zr}$ (a hard Lewis acid) at $75^{\circ} \mathrm{C}, \mathrm{pH} 8$, forming (2) cRGDY-PEG- $\left.{ }^{89} \mathrm{Zr}\right] \mathrm{C}^{\prime}$ dots. (b) Chelator-based strategy: DFO chelators are conjugated to the surface of amine-functionalized $\mathrm{NH}_{2}$-cRGDY-PEG- $\mathrm{C}^{\prime}$ dots by reacting DFO-NCS with the amine groups on the silica surface of the $\mathrm{C}^{\prime}$ dots. As synthesized (4) DFO-cRGDYPEG-C ${ }^{\prime}$ dots are then labeled with ${ }^{89} \mathrm{Zr}$ at $37{ }^{\circ} \mathrm{C}, \mathrm{pH} 7$, forming (5) ${ }^{89} \mathrm{Zr}$-DFO-cRGDYPEG-C ${ }^{\prime}$ dots. The molecular structures of the chelated radiometal for both strategies are rendered in 3D and 2D on the right. The atoms of silicon, oxygen, carbon, nitrogen, sulfur, hydrogen, and zirconium in the 3D renderings are colored in purple, red, gray, blue, yellow, white, and light green, respectively. 